International Journal of Modern Physics B (C) World Scientific Publishing Company

\title{
Electron-electron interaction effects on transport through mesoscopic superconducting hybrid junctions
}

\author{
ARIJIT SAHA * \\ Department of Physics, University of Basel \\ Klingelbergstrasse 82, CH-4056 Basel, Switzerland \\ Received Day Month Year \\ Revised Day Month Year
}

\begin{abstract}
Effects due to the proximity of a superconductor has motivated a lot of research work in the last several decades both from theoretical and experimental point of view. In this review we are going to describe the physics of systems containing normal metal superconductor interface. Mainly we discuss transport properties through such hybrid structures. In particular, we describe the effects of electron electron interaction on transport through such superconducting junction of multiple one-dimensional quantum wires. The latter can be described in terms of a non-Fermi liquid theory called Luttinger liquid. In this review, from the application point of view, we also demonstrate the possible scenarios for production of pure spin current and large tunnelling magnetoresistance in such hybrid junctions and analyze the influence of electron-electron interaction on the stability of the production of pure spin current.
\end{abstract}

Keywords: Quantum wires; Luttinger liquid; Superconductivity; Spintronics.

\section{Introduction}

In the past few decades, there has been an enormous amount of effort which has gone into designing one dimensional (1-D) quantum wires (QWs) in GaAs-AlGaAs hetero-structure. Other than the observation of quantization of conductance, the expectation is to observe signatures of non Fermi liquid behavior in the transport and other optical properties has also put a lot of motivation in designing 1-D QWs experimentally. To engineer 1-D QWs a new crystal growth technique has been developed, which tightly confines the electron on three sides by smooth semiconductor hetero-junctions. Such quantum wires are called cleaved edge overgrowth (CEO) quantum wire $(\mathrm{QW})^{1,2,3,4,5}$. The electron mean free path in these $\mathrm{QW}$ s can be as long as $10 \mu \mathrm{m}$. Also, experimentally measurable quantities in such QWs, are significantly affected by coulomb interactions between electrons inside them ${ }^{1}$. As long as one is interested in equilibrium phenomenon, the excitations contributing to any physical quantity are those excitations which are energetically close to the Fermi

*arijit.saha@unibas.ch 
energy $\left(E_{F}\right)$. Particularly in 1-D , excitations are around the Fermi points, where the dispersion can be linearized and hence, a large variety of models at low energies in 1-D belong to a special class of non Fermi liquid called Luttinger liquids (LL). The metallic state of this class of models is very different from that of usual Fermi liquid (FL) theory. There is no fermionic quasi-particle, and their elementary excitations are bosonic collective charge and spin fluctuations dispersing with different velocities. An incoming electron decays into such charge and spin excitations which then spatially separate with time (charge-spin separation). The correlations between these excitations are anomalous and show up as interaction-dependent non-universal power-law behaviour in many physical quantities whereas those of ordinary metals are characterized by universal (interaction independent) powers. The above mentioned salient features of LL have already been observed in cleaved edge overgrowth QWs ${ }^{1,2,3,4,5}$ and in carbon nanotubes ${ }^{31}$.

With the recent advancement in fabrication technology of semiconductor heterostructure it is now possible to study electronic transport in a variety of geometries of QWs among which the multi-point junctions is of special interest. Junction of several QWs has already been realized in Y-branched or multiple branched carbon nanotube ${ }^{6}$. Recent studies of ballistic transport through a QW have brought out the important role played by both scattering centers and the interactions between the electrons inside the QW. Theoretical studies using a real space renormalization group (RG) analysis show that repulsive interactions between electrons inside the QW tend to increase the effective strength of the impurity as one goes to longer and longer length scales ${ }^{7}$; experimentally, this leads to a decrease in the conductance as the temperature is reduced or the wire length is increased ${ }^{1,8,9,10,11}$. Considerable effort has also gone into understanding the effects of Fermi liquid leads ${ }^{12,13,14}$, multiple impurities ${ }^{15,16,17}$ and also contacts ${ }^{18}$ in two terminal measurements. Then, significant efforts have also gone into understanding the next logical step which is the effect of inter electrons interaction on the conductance of more complicated geometrical structures such as three or more QWs meeting at a junction. For the free electron case, the transport across the junction of multiple wires can be well understood under the framework of multi-terminal Landauer-Buttiker (LB) scattering theory. Although it is not straight forward to analyse transport through such junctions if electron-electron (e-e) interactions are also present inside the QW as LB formalism does not hold in this situation. This problem has been studied to some extent before in Ref. 19 and more recently in Ref. 20. To study this problem they use the technique of bosonization ${ }^{21,22,23,24,25}$ which can be used only for the weak and strong backscattering limits. On the other hand the problem of transport through a junction (characterized by an arbitrary scattering matrix $\mathbb{S}$ ) of $1-\mathrm{D}$ wires can be solved for any backscattering and in the weak e-e interaction limit, by using a RG technique introduced in Ref. 26. The main advantage of using this technique is the fact that one can access the intermediate fixed point which corresponds to scattering amplitudes with intermediate values ${ }^{27,28}$. Very recently, this RG method 
has also been generalized for QWs with arbitary e-e interactions ${ }^{29,30}$.

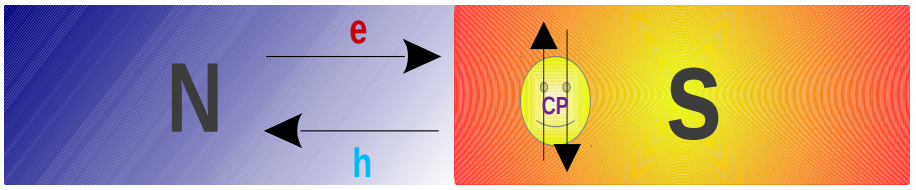

Fig. 1. (Color online) Cartoon of AR process in which an incident electron is reflected back from the NS interface as a hole and a Cooper pair transmits into the superconductor.

On the other hand, effects due to the proximity of a superconductor has motivated a lot of research work $32,33,34,35$ in the last several decades. A direct manifestation of proximity effect is the phenomenon of Andreev reflection (AR) which was predicted by Andreev in $1964^{32}$. In the case of a sufficientely clean normal-superconductor (NS) interface and a large superconducting gap the main contribution to transport comes from AR in which an incident electron, below the superconducting gap $\Delta$ is reflected back as a hole from the interface and a Cooper pair (charge of 2e) jump into the superconductor (see also Fig. 1). In the AR processes the pairing amplitude of the superconductor is induced in the normal-metal side, while the attractive interaction potential between electrons is identical to zero in the normal conductor. An even more intriguing example where the proximity effect manifests itself is the phenomenon of crossed Andreev reflection (CAR) which can only take place in a normal metal-superconductor-normal metal (NSN) junction provided the distance between the two normal metals is less than or equal to the phase coherence length of the superconductor. This is a nonlocal process where an incident electron from one of the normal metal leads pairs up with another electron from the other lead to form a Cooper pair ${ }^{40,41}$ and jumps into the superconductor. The signature of this nonlocal process also has been verified by a number of recent experiments involving NSN junction ${ }^{36,37,38,93}$ and also carbon nanotubes ${ }^{39}$. Also the relevance of CAR regarding production of entangled electron pairs in nano devices for quantum computation has attracted a lot of attention in recent times $42,43,44,45,46,47,48,49,50,51,106,107$. Non-local entangled electron pairs involving NSN junction can be realized in a Cooper pair beam splitter ge- 
ometry $53,54,55,56,94$ in which such pairs are produced via CAR processes when the superconductor is biased with respect to the normal leads comprising the junction. Very recently, conclusive evidence of extremely high efficiency Cooper pair splitting via observing positive two-particle cross correlations of the shot noise of the split electrons via CAR process in the beam splitter geometry, has been put forwarded both theoretically ${ }^{52}$ and experimentally 57,114 .

In recent times, a lot of attention has also gone into understanding the effects of e-e interactions on AR processes in case of NS junctions in the context of 1-D quantum wires ${ }^{58,59,60,61,64}$, quantum dots ${ }^{95,96,97,98}$, carbon nanotubes ${ }^{62}$ and proximity effects in LL ${ }^{63}$. The power law dependence of the Andreev conductance for the NS junction case was first obtained using weak interaction renormalization group (WIRG) approach by Takane and Koyama in Ref. 58. This was in agreement with earlier results from bosonisation ${ }^{59}$, which, however, could only handle perturbative analysis around the strong back-scattering (SBS) and weak back-scattering (WBS) limits. The WIRG approach, on the other hand, can study the full cross-over from WBS limit to SBS limit. Hence the WIRG approach is very well-suited for studying problems where the aim is to look for non trivial fixed points with intermediate transmissions and reflections. The latter would be difficult using a bosonisation approach. For the NS junction case, it was also shown that the power law exponent for the temperature dependence of conductance was twice as large as the exponent for a single barrier in a QW. This happens due to the existence of AR process in which both electron and hole channels take part in transport.

The organization of the rest of the review is as follows. In Sec. 2 we give a generic description of the model for junction of 1-D QWs which are described by the LL theory. In particular in Sec. 2.2 we describe the WIRG method applied to model the e-e interactions in 1-D QWs and a comparative analysis between WIRG method and Bosonization is presented in Sec. 2.3. In Sec. 3.1 we analyse the superconducting junction of many $(N \geq 2) 1-\mathrm{D}$ QWs by the WIRG procedure and in (Sec. (3.1.13.1.4)) we present the RG fixed point analysis of some special geometry. In Sec. 3.2 we discuss the results of RG flows for the conductance in different geometries comprising of superconducting junction. Our study reveals the striking fact that due to the inter-play of the proximity and the interaction effects, one gets a novel nonmonotonic behavior (non LL behavior) of conductance for the case of NSN junction as a function of the temperature. In Sec. 4 we study the stability analysis of the RG flow for the NSN junction of LL wires. In particular, we compute the power laws associated with the RG flow around the various fixed points of this system and also obtain the power law dependence of linear response conductance on voltage bias or temperature around them. In Sec. 5 we discuss the possible applications of different superconducting junctions of 1-D QWs and in particular we show that one can have pure spin current (SC) and large tunneling magnetoresistance (TMR) in such geometries. We also analyze the influence of e-e interaction and see how it stabilizes or de-stabilizes the production of pure SC. Finally in Sec. 6, we present 
our summary and possible outlooks of the topic.

\section{General model for junction}

The model for the junction essentially comprises of $N$ semi-infinite QWs meeting at a point. The QWs are parameterized by the coordinates $x_{i}, i=1,2, \ldots, N$. The junction is the point where the $x_{i}$ are simultaneously equal to 0 . The convention is, each $x_{i}$ increases from 0 as one goes outwards from the junction along wire $i$. The incoming and outgoing single particle wave functions on wire $i$ are denoted by $\psi_{I i}\left(x_{i}\right)$ and $\psi_{O i}\left(x_{i}\right)$ respectively (ignoring the spin label $\sigma$ for the time being); see Fig. 2. For a given wave number $k>0$, these wave functions are proportional to the plane waves $\exp \left(-i k x_{i}\right)$ and $\exp \left(i k x_{i}\right)$.

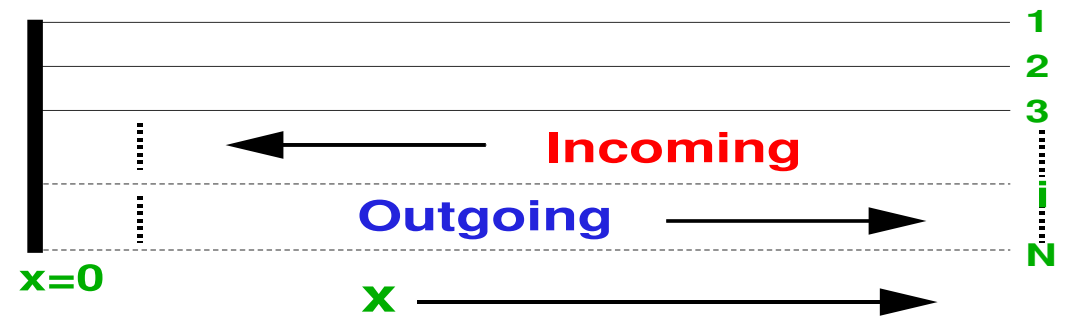

Fig. 2. (Color online) Cartoon of $N$ QWs meeting at a junction at $x=0$. Here the figure also illustrates the incoming and outgoing wave directions to and from the junction. The junction is shown as an extended line for better visibility but actually it's a point.

The coefficients of the plane waves are related to each other by a $N \times N$ scattering matrix denoted by $\mathbb{S}$. Denoting the incoming and outgoing wave functions at the junction by the columns $\psi_{I}(0)$ and $\psi_{O}(0)$ respectively, we have the relation

$$
\psi_{O}(0)=\mathbb{S} \psi_{I}(0)
$$

$\mathbb{S}$ must be unitary for current conservation and in the absence of a magnetic field, the $\mathbb{S}$ matrix must be symmetric also so that it respects time reversal symmetry. The diagonal entries of $\mathbb{S}$ are the reflection amplitudes $r_{i i}$, while the off-diagonal entries are the transmission amplitudes $t_{i j}$ to go from wire $j$ to wire $i$. 


\section{1. $\quad$ Model for electron - electron interaction}

We start with the description of a single QW for the moment, so that the label $i$ can be dropped. At low temperatures or at low-energy, excitations are dominated by modes near the Fermi points $\pm k_{F}$ in 1 -D QW. Around the $\pm k_{F}$ the secondquantized Fermi field $\Psi(x)$ corresponding to these modes can be expanded in terms of the scattering basis as given below

$$
\Psi(x)=\Psi_{I}(x) e^{-i k_{F} x}+\Psi_{O}(x) e^{i k_{F} x},
$$

It is important to note that, the fields $\Psi_{I}$ and $\Psi_{O}$ defined in Eq. 2 vary slowly on the scale of the inverse Fermi momentum $k_{F}^{-1}$, since we have separated out the rapidly varying functions $\exp \left( \pm i k_{F} x\right)$. Henceforth we use the notation $\Psi_{I}$ and $\Psi_{O}$ for these slowly varying second-quantized fields, rather than the incoming and outgoing fields defined earlier. For such fields, we will only be interested in Fourier components with momenta $k$ which satisfy $|k|<<k_{F}$. Hence we can linearize the dispersion relations such that the spectrum takes the form, $E= \pm \hbar v_{F} k$ for the fields $\Psi_{O}$ and $\Psi_{I}$ respectively, where $v_{F}$ is the Fermi velocity. Also it is practical to assume that the elements of $\mathbb{S}$-matrix are energy independent i.e. independent of $k$ for small $k$.

The e-e interaction part of the hamiltonian can be written as

$$
H_{\text {int }}=\frac{1}{2} \iint d x d y \rho(x) V(x-y) \rho(y)
$$

where $V(x)$ is a real quantity and function of $x$.

The density $\rho$ is given in terms of the fermion fields as $\rho(x)=\Psi^{\dagger}(x) \Psi(x)$. Using Eq. 2, we have

$$
\begin{aligned}
\rho(x)= & \Psi_{I}^{\dagger} \Psi_{I}+\Psi_{O}^{\dagger} \Psi_{O} \\
& +\Psi_{I}^{\dagger} \Psi_{O} e^{i 2 k_{F} x}+\Psi_{O}^{\dagger} \Psi_{I} e^{-i 2 k_{F} x} .
\end{aligned}
$$

Again let us assume a highly screened short-ranged interaction for $V(x-y)$ in Eq. 3 such that the arguments $x$ and $y$ of the two density fields can be set equal to each other wherever possible. In doing so, we neglect terms with scaling dimension greater than 2, and are therefore irrelevant under RG flow. Using the anti-commutation relations between different fermion fields, we obtain

$$
H_{\mathrm{int}}=g_{2} \int d x \Psi_{I}^{\dagger} \Psi_{I} \Psi_{O}^{\dagger} \Psi_{O},
$$

where $g_{2}$ is related to the Fourier transform of $V(x)$ as $g_{2}=\tilde{V}(0)-\tilde{V}\left(2 k_{F}\right)$ for the spinless electrons. It is interesting to note that $g_{2}$ is zero if $V(x)$ is a $\delta$-function potential as in that case $\tilde{V}(0)=\tilde{V}\left(2 k_{F}\right)$ for the spinless case. This argument reveals the fact that an ultra-short range interaction like the $\delta$-function potential is not useful. Thus the interaction may be short-ranged, but must have some finite range. Different QWs may have different values of the interaction parameter $g_{2}$ represented 
by $g_{2 i}$. For later use, we define the dimensionless constants as

$$
\alpha_{i}=\frac{g_{2 i}}{2 \pi \hbar v_{F}},
$$

where we assume that the Fermi velocity $v_{F}$ is the same on all wires. For 1-D interacting electrons (LL theory) the most efficient and popular approach to analyze transport behaviour is to implement bosonization formalism ${ }^{21,22,23,24,25}$. For spinless fermions, the bosonic theory is characterized by two parameters, $v$, the renormalized Fermi velocity and a dimensionless parameter $K$ which parameterizes the strength of inter electron interactions inside the QWs. Typically, $K$ governs the exponents which appear in the power-law fall-offs of various correlation functions in the theory. For a model defined on the entire real line with the interaction parameter $g_{2}$ or $\alpha$ defined above, we find that ${ }^{25}$

$$
K=\left(\frac{1-\alpha}{1+\alpha}\right)^{1 / 2} .
$$

Thus $K=1$ corresponds to the noninteracting fermions (Fermi liquid theory), while $K<1$ and $K>1$ to short-ranged repulsive and attractive interactions respectively. For weak interactions, we see that $v=v_{F}$ while $K=1-\alpha$ to first order in $\alpha$. In this review, we will be interested in the case in which the e-e interaction is weak and repulsive, i.e., the parameters $\alpha_{i}$ are all positive and small.

\subsection{Weak interaction renormalization group approach}

In this sub section we introduce the weak interaction renormalization group (WIRG) method which was first developed by Yue et al. ${ }^{26}$ for the case of junction of two QWs and then extended by Lal et al. ${ }^{27}$ to the case of junction of multiple QWs. In comparison to Bosonization, this method is instructive and physically transparent. Using this method, RG equations for all the $\mathbb{S}$-matrix elements can be evaluated to leading order in $\alpha$. The basic idea behind this method is the following

In the presence of a non-zero reflection amplitude $r_{i i}$, the density of noninteracting fermions in wire $i$ gives rise to Friedel oscillations with wavenumber $2 k_{F}$. In presence of weak e-e interaction inside the QW, an electron scatters not only from the junction but also from these Friedel oscillations by an amount proportional to the parameter $\alpha_{i}$. Yue et al. use this idea to derive the RG equations for an arbitrary $\mathbb{S}$-matrix located at the junction of two semi-infinite QWs. In the limits of both weak back scattering $\left(r_{11} \rightarrow 0\right)$ and strong back scattering $\left(\left|r_{11}\right| \rightarrow 1\right)$, their results reduce to those known from bosonization ${ }^{7,21,22}$.

Here we first derive the form of the density oscillations in one particular QW given that there is a reflection coefficient $r$ for incoming electron waves along that wire. If the momentum of the incident electron is in the vicinity of Fermi momentum $k_{F}$, we can write the wave function in the form

$$
\psi_{k}(x)=e^{-i\left(k+k_{F}\right) x}+r e^{i\left(k+k_{F}\right) x},
$$


where $|k|<<k_{F}$. In the ground state of the noninteracting system, the density is given by

$$
<\rho(x)>=\int_{-\infty}^{0} \frac{d k}{2 \pi} \psi_{k}^{\star}(x) \psi_{k}(x),
$$

where we use the fact that only states with energy less than $E_{F}$ (i.e., momenta less than $k_{F}$ ) are occupied, and we extend the lower limit of Eq. 9 to $-\infty$ for convenience. (Alternatively, we can impose a cut-off at the lower limit of the form $\exp (\epsilon k)$, and take the limit $\epsilon \rightarrow 0$ at the end of the calculation). We then find that $\rho$ has a constant piece $\rho_{0}$ (which can be eliminated by normal ordering the density operator), and a $x$-dependent piece given by

$$
<\rho(x)>-\rho_{0}=\frac{i}{4 \pi x}\left(r^{\star} e^{-i 2 k_{F} x}-r e^{i 2 k_{F} x}\right) .
$$

Using the expression in Eq. 4, we find that the expectation value $<\Psi_{I}^{\dagger} \Psi_{I}+\Psi_{O}^{\dagger} \Psi_{O}>$ is a constant, while

$$
\begin{aligned}
& <\Psi_{O}^{\dagger} \Psi_{I}>=\frac{i r^{\star}}{4 \pi x}, \\
& <\Psi_{I}^{\dagger} \Psi_{O}>=-\frac{i r}{4 \pi x} .
\end{aligned}
$$

Note that there is also a contribution to $\rho(x)$ from the waves transmitted from the other wires; however those are independent of $x$ and can be absorbed in the constant piece $\rho_{0}$. Hence, the Friedel oscillations given by Eq. 10 in a given QW arise only from reflections within that wire. Also here the energy dependence of the $\mathbb{S}$-matrix elements has been neglected as only excitations $|E|<<E_{F}$ has been taken into account. Later WIRG with the explicit energy dependence of the $\mathbb{S}$-matrix elements in case of resonant tunneling in LL wires has been analysed in Ref. [28, 88].

Next we derive the amplitude of scattering of the fermions from the Friedel oscillations, using a Hartree-Fock decomposition of the interaction in Eq. 5. The reflection is caused by the following terms in the decomposition

$$
\begin{aligned}
H_{\mathrm{int}} & =-g_{2} \int_{0}^{\infty} d x\left(<\Psi_{O}^{\dagger} \Psi_{I}>\Psi_{I}^{\dagger} \Psi_{O}+<\Psi_{I}^{\dagger} \Psi_{O}>\Psi_{O}^{\dagger} \Psi_{I}\right), \\
& =-\frac{i g_{2}}{4 \pi} \int_{0}^{\infty} \frac{d x}{x}\left(r^{\star} \Psi_{I}^{\dagger} \Psi_{O}-r \Psi_{O}^{\dagger} \Psi_{I}\right),
\end{aligned}
$$

where we have used Eq. 11 to write the second line in Eq. 12. Now we derive the amplitude to go from a given incoming wave with momentum $k$ to an outgoing wave (or vice versa) under the action of $\exp \left(-i H_{\text {int }} t\right)$. The amplitude can be written as

$$
\begin{aligned}
-i \int \frac{d k^{\prime}}{2 \pi} 2 \pi \delta\left(E_{k}\right. & \left.-E_{k^{\prime}}\right) \mid \text { outgoing, } k^{\prime}> \\
& \times<\text { outgoing, } k^{\prime}\left|H_{\mathrm{int}}\right| \text { incoming, } k> \\
=\mid \text { outgoing, } k> & \frac{i g_{2} r}{4 \pi \hbar v_{F}} \int_{0}^{\infty} \frac{d x}{x} e^{-i 2 k x},
\end{aligned}
$$


where we have used Eq. 12, the dispersion relation $E_{k}=\hbar v_{F} k$ (such that $\delta\left(E_{k}-\right.$ $\left.\left.E_{k^{\prime}}\right)=\left(1 / \hbar v_{F}\right) \delta\left(k-k^{\prime}\right)\right)$, and the wave functions $\exp ( \pm i k x)$ of the outgoing and incoming waves respectively to arrive at Eq. 13. The integral over $x$ in Eq. 13 is divergent at the lower end; we therefore introduce a short-distance cut-off $d$ which is the inter particle spacing here. The amplitude in Eq. 13 then reduces to

$$
-\frac{\alpha r}{2} \ln (k d) \text {. }
$$

plus contributions which remain finite as $k d \rightarrow 0$; we have used Eq. 6 here. Similarly, the amplitude to go from an outgoing wave to an incoming wave due to the scattering from Friedel oscillations is given by

$$
\frac{\alpha r^{\star}}{2} \ln (k d)
$$

These reflections from the Friedel oscillations can then be combined along with the

(a)

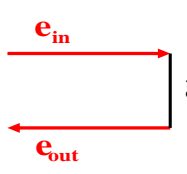

(b)

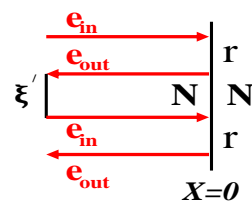

(c)

\begin{tabular}{ll|ll}
$\mathbf{e}_{\text {in }}$ & $\mathbf{t}$ & $\mathbf{e}_{\text {out }}$ \\
& $\mathbf{N}$ & $\mathbf{N}$ & \\
$\mathbf{e}_{\text {out }}$ & $\begin{array}{c}\mathrm{t} \\
\boldsymbol{X}=\boldsymbol{O}\end{array}$ & $\mathbf{e}_{\text {in }}$
\end{tabular}$\xi^{\prime}$

Fig. 3. (Color online) The processes that contribute to the amplitude for an incoming electron to transform to an outgoing electron. Note that all the processes shown here are to first order in the interaction parameters since they only involve a single scattering from a Friedel oscillation. Process (a) involves scattering from the Friedel oscillation before the electron reaches the junction. Process (b) involves two reflections from the junction along with a scattering from the Friedel oscillation in the same wire. On the other hand process (c) involves two transmissions through the junction and a scattering from the Friedel oscillation in the other wire. In the diagrams, $\xi=-\frac{\alpha r}{2} \ln (k d)$ and $\xi^{\prime}=\frac{\alpha r^{\star}}{2} \ln (k d)$.

bare $\mathbb{S}$-matrix at the junction to calculate the corrections to it. For instance, let us consider $r_{i i}$ at first. To first order in the interaction parameters $\alpha_{i}$, this amplitude gets corrections from the following processes. An incoming electron wave on wire $i$ can either (i) turn into an outgoing electron on the same wire with the amplitude given in Eq. 14 (with $r$ replaced by $r_{i i}$ in that expression), or (ii) get reflected from the junction with amplitude $r_{i i}$ thereby turning into an outgoing wave, turn back 
into an incoming wave after scattered back from the Friedel oscillations according to Eq. 15, then get reflected again from the junction, or (iii) transmit through the junction into wire $j$ (with $j \neq i$ ) with amplitude $t_{j i}$, turn from an outgoing wave to an incoming wave on wire $j$ according to Eq. 15 (with $r$ replaced by $r_{j j}$ ), then transmit back through the junction to wire $i$ with amplitude $t_{i j}$. The above mentioned three processes (see Fig. 3) effectively give correction to the bare $r_{i i}$ and the renormalized $r_{i i}$ therefore becomes

$$
\begin{aligned}
& d r_{i i}=-A_{i i} \ln (k d), \\
& \text { where } A_{i i}=-\frac{1}{2}\left[-\alpha_{i} r_{i i}+\alpha_{i}\left|r_{i i}\right|^{2} r_{i i}\right. \\
& \left.+\sum_{j \neq i} \alpha_{j} t_{i j} r_{j j}^{\star} t_{j i}\right] .
\end{aligned}
$$

Similarly, we calculate the correction to the transmission $t_{j i}$ from wire $i$ to wire $j$ and it is given by

$$
\begin{aligned}
& d t_{j i}=-A_{j i} \ln (k d), \\
& \text { where } \quad A_{j i}=-\frac{1}{2}\left[\alpha_{i} t_{j i}\left|r_{i i}\right|^{2}+\alpha_{j}\left|r_{j j}\right|^{2} t_{j i}\right. \\
&\left.\quad+\sum_{k \neq i, j} \alpha_{k} t_{j k} r_{k k}^{\star} t_{k i}\right] .
\end{aligned}
$$

Hence, one can derive the RG equations for the $\mathbb{S}$-matrix which is considered to be a function of a length scale $L$; by replacing $-\ln (k d)$ in Eqs. (16-17) by $d l$, where $l=\ln (L / d)$. The RG equations therefore take the form

$$
\begin{aligned}
& \frac{d r_{i i}}{d l}=A_{i i}, \\
& \frac{d t_{i j}}{d l}=A_{i j},
\end{aligned}
$$

where $A_{i i}$ and $A_{i j}$ are given by Eq. 16 and Eq. 17 respectively. We can also write Eqs. 18 in a simpler way. Given the matrix $\mathbb{S}$ and the parameters $\alpha_{i}$ (which do not flow under RG for the spinless case), we define a diagonal matrix $F$ whose entries are

$$
F_{i i}=-\frac{1}{2} \alpha_{i} r_{i i}
$$

Then the RG equations for the $\mathbb{S}$-matrix can be written in the matrix form as

$$
\frac{d \mathbb{S}}{d l}=\mathbb{S} F^{\dagger} \mathbb{S}-F .
$$

Note that the derivation of the RG equation for the $\mathbb{S}$-matrix presented above is correct only when the e-e interaction strength inside the $\mathrm{QW}$ is perturbative i.e. first order in $\alpha$. A non-perturbatibe (arbitary strength of $\alpha$ ) RG approach has been developed recently by ${ }^{29,30}$ to study transport of interacting electrons through a potential barrier. In their work they calculate the linear response conductance of electrons in a LL with arbitary e-e interaction strength. Their result also agrees well with the known limiting cases of WIRG ${ }^{26,27}$. 


\subsection{WIRG vis-a-vis Bosonisation for junction}

In this subsection we present a comparative discussion between Bosonization and WIRG for analyzing transport through a quantum scatterer (for instance, a simple static barrier or a dynamical magnetic impurity like Kondo spin) in a 1-D QW. The latter is qualitatively different from its higher dimensional counterpart ${ }^{7}$ as in $1-\mathrm{D}$, due to e-e interactions, the Fermi-liquid ground state is destroyed and the electrons form a non-Fermi liquid ground state known as Luttinger Liquid ${ }^{66}$. The low energy dynamics of such 1-D system is governed mainly by coherent particlehole excitations around the left and the right Fermi points. Hence, it is natural to use bosonic fields to describe these low lying excitations. The later can be implemented by re-expressing the original fermions using boson coherent state representation ${ }^{21,22,24,25}$ which is referred to as bosonisation. Although the bosonization approach only allows for a perturbative analysis for transport around the limiting cases of SBS and WBS for the quantum impurity problem. On the other hand, if we start with a weakly interacting electron gas, it is possible to do a perturbative analysis in the e-e interaction strength around the free fermion Hamiltonian, but treating the strength of the quantum impurity exactly. This approach allows us to study transport through the impurity for any scattering strength. The advantage of this approach lies in the fact that even in presence of e-e interaction, one can use single particle pictures such as the transmission and reflection amplitudes ( $\mathbb{S}$ matrix) in order to characterize the impurity. Then the idea is to calculate correction to the transmission and reflection amplitudes perturbatively in the e-e interaction strength.

Now in the next step, since we are working in 1-D, the perturbative correction turns out to be logarithmically divergent. To obtain a finite result, one has to sum up all such divergent contributions to the transmission and reflection amplitudes to all relevant orders at a given energy scale. This was first done by Matveev et al. in Refs. 67 and 26 in the context of a single (scalar) scatterer for both spinless and spin-full electrons using the "poor man's scaling" approach ${ }^{69}$. For the spin-less case, it was shown that the logarithmic correction to the bare transmission probability (to first order in interaction parameter parameterized by $\alpha$ ) was $\delta T=2 \alpha T_{0}\left(1-T_{0}\right) \ln (k d)$ and the explicit RG equation for transmission probability was $d T / d l=-2 \alpha T(1-T)$ where $k$ is the momentum of the fermion measured from $k_{F}, d$ is a short distance cut-off and $\alpha$ is the e-e interaction parameter given by $\alpha=\alpha_{1}-\alpha_{2}$ with $\alpha_{1}=V(0) / 2 \pi \hbar v_{F}$ and $\alpha_{2}=V\left(2 k_{F}\right) / 2 \pi \hbar v_{F}$.

The RG equation upon integration gives rise to final answear for the transmission probability which can be written as,

$$
T(L)=\frac{T_{0} e^{-2 \alpha l}}{\left[1-T_{0}+T_{0} e^{-2 \alpha l}\right]}=\frac{T_{0}\left(\frac{d}{L}\right)^{2 \alpha}}{\left[1-T_{0}+T_{0}\left(\frac{d}{L}\right)^{2 \alpha}\right]},
$$

where, $l=-\ln (k d)=\ln (L / d)$ and $L$ is the length of the QW. Also $l$ can also be measured as a function of the temperature by introducing the thermal length, 
$L_{T}=\left(\hbar v_{F}\right) /\left(k_{B} T\right)$. In Eq. $21 T_{0}$ is the bare transmission probability at the short distance cut-off, $d$. It is easy to see from Eq. 21 that for very small values of $T_{0}$, the latter can be neglected in the denominator of the expression for $T(L)$ leading to a pure power law scaling behavior consistent with the power law known from bosonisation in the WBS limit. Similarly for the spin-full electrons, it can be shown that the parameter $\alpha$ in the power law gets replaced by a new parameter, $\beta$ given by $\beta=\left(g_{2}-2 g_{1}\right) / \pi \hbar v_{F}$ where $g_{2}=g_{2}(k)$ and $g_{1}=g_{1}(k)$ are momentum dependent functions or 'running coupling constants' which is in sharp contrast to the spin less case. At high momentum, (or equivalently, at the short distance cut-off scale), $g_{1}(d)=V\left(2 k_{F}\right)$ and $g_{2}(d)=V(0)$. Due to the presence of the extra logarithmic dependence originating from scaling of the interaction parameter itself (see Eq. 23 and Eq. 24 below), the expression for transmission probability ${ }^{26,67}$, no longer follows a pure power law scaling even for small values of $T_{0}$. Instead $T(L)$ is now given by

$$
T(L)=\frac{\left[T_{0}\left[1+\alpha_{1} \ln \left(\frac{L}{d}\right)\right]^{\frac{3}{2}}\left(\frac{d}{L}\right)^{\left(2 \alpha_{2}-\alpha_{1}\right)}\right]}{\left[1-T_{0}+T_{0}\left[1+2 \alpha_{1} \ln \left(\frac{L}{d}\right)\right]^{\frac{3}{2}}\left(\frac{d}{L}\right)^{\left(2 \alpha_{2}-\alpha_{1}\right)}\right]},
$$

using the length scale dependence of $g_{1}(L)$ and $g_{2}(L)$ given by 68

$$
\begin{aligned}
& g_{1}(L)=\frac{V\left(2 k_{F}\right)}{\left[1+\frac{V\left(2 k_{F}\right)}{\pi v_{F}} \ln \left(\frac{L}{d}\right)\right]}, \\
& g_{2}(L)=V(0)-\frac{1}{2} V\left(2 k_{F}\right)+\frac{1}{2} \frac{V\left(2 k_{F}\right)}{\left[1+\frac{V\left(2 k_{F}\right)}{\pi v_{F}} \ln \left(\frac{L}{d}\right)\right]} .
\end{aligned}
$$

Note that in the absence of e-e interaction induced back-scattering (i.e., when $\left.V\left(2 k_{F}\right)=0\right)$, there is no correction to the power law behavior. Hence, bosonisation, which ignores e-e back-scattering always results in power law behaviour. Although, when e-e interaction induced back-scattering is included, the sign of $g_{2}-2 g_{1}$ can change under RG flow, and hence, there can be a qualitative change in the behavior of the conductance. The latter actually develops a non-monotonic dependence on the temperature; it first grows and then drops to zero as one approaches very low temperature. Although, except for this non-monotonic behavior of conductance for the spin-full case, there is no new physics which is achieved by studying the full crossover from WBS to SBS. In conclusion, both bosonisation and WIRG methods predict that for the single impurity (scalar) problem there are only two fixed points(a) the perfectly back-scattering (no transmission) case is the stable fixed point and (b) the absence of back-scattering (perfect transmission) case is the unstable fixed point. There are no other fixed points with intermediate reflection and transmission.

It is first shown by Lal et al. in Ref. 27, using the WIRG approach that even though there are only two fixed points for the two-wire-junction, surprisingly enough, the three-wire-junction has a host of fixed points, some of which are isolated fixed points while others are one parameter or multi parameter families of fixed points. It is also shown to be true for more than three wires. From this point of view, 
the physics of a two-wire-junction is different from its three-wire-counterpart. Also in Ref. 27, it is shown that for a multiple-wire-junction, the RG equations for the full $\mathbb{S}$-matrix characterizing the junction take a very convenient matrix form shown in Eq. 20. The advantage of writing the RG equation in this way is that it immediately facilitates the hunt for various fixed points. All one needs to do is to set the matrix on the LHS of Eq. 20 to zero. The latter scheme formally provides us with all the fixed points associated with a given $\mathbb{S}$-matrix. The three-wire-junction was also studied using bosonisation and conformal field theory methods in the context of fixed point structure and conductance around them $19,20,78,79,80,81,82,91$, tunneling density of states ${ }^{89}$ and power dissipation ${ }^{90}$. The latter approaches confirmed some of the fixed points found using WIRG. It also gave some extra fixed points which were related to charge fractionalisation at the junction, and which could not be seen within the WIRG approach. Very recenly, tunneling density of states ${ }^{83}$ and transport properties of three-wire-junction comprising of normal or chiral LL wires have been studied using the WIRG method with arbitary repulsive e-e interaction strength and different interaction strength in each QW ${ }^{84,85,86,87}$. In their work they predict a new M (mystery) fixed point at which the conductance takes an intermediate value and also this $\mathrm{M}$ fixed point becomes stable for attractive e-e interaction. Their results also match with the bosonization treatment of three-wire geometry ${ }^{20}$ and Ref. 27 in the weak interaction limit.

The WIRG method was further extended to more complicated geometries made out of junctions of QW which can host resonances and anti-resonances in Ref. 71 . The scaling of the resonances and anti-resonances were studied for various geometries which included the ring and the stub geometry. This approach was further extended in Refs. [72, 73] to study the multiple-wire-junction with a dynamical scatterer, i.e. a (Kondo) spin degree of freedom. The coupled RG equations involving the Kondo couplings, $J_{i j}$ as well as the $\mathbb{S}$-matrices were solved. For different starting scalar $\mathbb{S}$-matrices, the RG flows of the Kondo couplings was studied. The temperature dependence of the conductances was shown to have an interesting interplay of the Kondo power laws as well as the interaction dependent power laws.

Almost a decade ago, the WIRG method was also extended to the case of NS junction ${ }^{58,59}$. In the vicinity of the superconductor, it is well-known that the system is described by holes as well as electrons ${ }^{34}$. Hence the $\mathbb{S}$-matrix characterizing the junction not only includes the electron channel but also the hole channel. Hence, both electron and hole channels take part in transport. Naively, one might expect that in the presence of particle-hole symmetry, the only effect of including the hole channel would be to multiply the conductance by a factor of two (in analogy with inclusion of spin and imposing spin up-spin down symmetry). However, it was shown ${ }^{58,59}$ that in the vicinity of a superconductor, the proximity induced scattering potential that exists between electron and holes, also gets renormalized by e-e interactions. When this scattering is also taken into account, the correction to the scattering amplitude to first order in the interaction parameter depends on $\left(2 g_{2}-g_{1}\right)$ instead of $\left(g_{2}-2 g_{1}\right)$ in the spinful case. It is worth stressing that this par- 
ticular linear combination of the interaction parameters $\left(g_{i}{ }^{\prime}\right.$ 's) is independent of the scaling as the logarithmic factors $(l=\ln (k d))$ in Eqs. 23 and 24 cancel each other. Hence, there is no non-monotonic behavior of the conductance in this scenario. The WIRG predicted only two fixed points, the Andreev fixed point (perfect AR) which turns out to be an unstable one and the perfectly reflecting fixed point which is the stable fixed point. The NS junction has also been studied using bosonisation ${ }^{74}$. It is easy to check that the power laws resulting from bosonisation agree with those obtained from the WIRG, when the e-e interaction induced back-scattering (which is neglected in the bosonisation method) is ignored.

In the next section, we apply the WIRG method to the superconducting junction of multiple 1-D quantum wires ${ }^{75}$. We note that we now have two complications (a) multiple wires are connected to the junction and (b) we have both electron and hole channels associated with the junction. So in this case, even for the NS junction, we have two spin channels as well as the electron and hole channels, so the scattering matrix is four component. For $N$ wires, the scattering matrix is $4 N \times 4 N$ dimensional. Although, we expect our method to work well even in this case, there is one caveat we must keep in mind. We have incorporated the effect of the superconductor as a boundary condition on the QW and neglected any internal dynamics of the superconductor itself. This should work reasonably well as long as we are studying transport at energies much below the superconducting gap. Our main result here is that the conductance across the junction depends on both $g_{1}$ and $g_{2}$ and not on a a special combination $2 g_{2}-g_{1}$ (as in NS case) which does not get renormalized under RG flow. Hence, the cancellation of the logarithmic terms in the effective interaction parameter is specific to the NS case and is not true in general. For $N \geq 2$ wires attached to a superconductor, we expect a non-monotonic form of the conductance. We also expect to get a host of fixed points with intermediate transmission and reflection, knowledge of which can be of direct relevance for spintronics ${ }^{76}$ and application to device fabrication of such geometries.

\section{Superconducting junction of multiple 1-D quantum wires}

In this section, let us consider multiple $(N)$ quantum wires meeting at a junction on which a superconducting material is deposited on top of it as depicted in Fig. 4. The wires are parameterized by coordinates $x_{i}$, with the superconducting junction assumed to be at $x_{i}=0$. We consider a situation where the effective width ' $a$ ' of the superconductor between two consecutive wires is of the order of the phase coherence length of the superconductor (size of the Cooper pair). For our purpose, it is safe to ignore the finiteness of ' $a$ ' and effectively treat the junction of QW as a single point in space with an appropriate boundary condition called Andreev boundary condition. We parameterize the junction by the following quantum mechanical amplitudes via a $\mathbb{S}$-matrix. There are two kinds of reflection amplitudes: the normal reflection amplitude $\left(r_{i i s_{i} s_{i}}\right)$ and the AR amplitude $\left(r_{A i i s_{i} s_{i}}\right)$ on each QW. In addition, there are two kinds of transmission amplitudes between different wires whic 


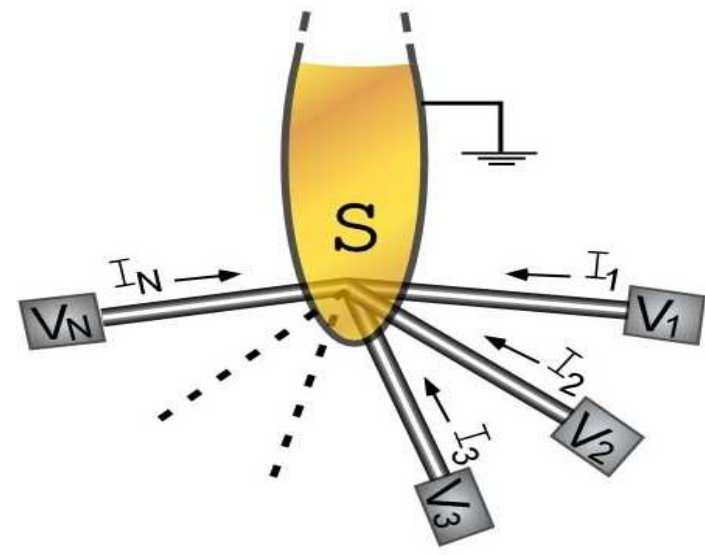

Fig. 4. (Color online) Multiple QWs connected to a superconducting junction. The dashed lines represents the fact that the model can be trivially extended to more than two wires. ' $a$ ' is the effective length of the superconductor which is assumed to be of the order of phase coherence length of it. Figure adapted from Ref. 75.

are the CT amplitude $\left(t_{i j s_{i} s_{j}}\right)$ and the non-local CAR amplitude $\left(t_{A i j s_{i} s_{j}}\right)$. The indices $s_{i}, s_{j}$ refer to the spin of incoming and outgoing particles. As we consider a singlet superconductor at the junction, spin remains conserved in all the processes mentioned above. Thus, the boundary conditions can be parametrized by a $4 N \times 4 N$ scattering matrix for $N$ QWs connected to the superconducting junction.

Let us now consider the various symmetries that can be imposed to simplify the $4 N \times 4 N$ S-matrix at the junction. We impose particle-hole symmetry, i.e., we assume that the reflection and transmissions are the same for particles (electrons) and holes. Further, in the absence of a magnetic field, spin symmetry is conserved which implies that the various transmission and reflection amplitudes for spin up-down electrons and holes are equal. (This spin symmetry breaks down in the presence of magnetic fields, or in the case of ferromagnetic wires where the spin is fully polarized). Also, since we assume that all the wires, connected to the superconductor, are indistinguishable, we can impose a wire index symmetry. (This symmetry again can be broken if we take some ferromagnetic and some normal wires attached to the superconductor). On imposing these symmetries, the $\mathbb{S}$-matrix for a two-wire system with the superconductor at the junction is given by

$$
\mathbb{S}=\left[\begin{array}{cc}
\mathbb{S}_{\uparrow} & 0 \\
0 & \mathbb{S}_{\downarrow}
\end{array}\right]
$$


with

$$
\mathbb{S}_{\uparrow}=\mathbb{S}_{\downarrow}=\left[\begin{array}{cccc}
r & t & r_{A} & t_{A} \\
t & r & t_{A} & r_{A} \\
r_{A} & t_{A} & r & t \\
t_{A} & r_{A} & t & r
\end{array}\right],
$$

Here $r$ stands for normal reflection of electron or hole in each wire, and $r_{A}$ represents AR from electron to hole or vice-versa in each wire. On the other hand $t$ represents the elastic CT amplitude $\left(t=t_{12}=t_{21}\right)$ while $t_{A}$ represents the CAR amplitude $\left(t_{A}=t_{A 12}=t_{A 21}\right)$ from one wire to the other. For the spin symmetric case, there are two such matrices, one for spin up electrons and holes and the other one for spin down electrons and holes. Note that this is the relevant $\mathbb{S}$-matrix at energy scales (temperature and applied voltage on the wires) $k_{B} T, e V_{i} \ll \Delta$, where $\Delta$ is the bulk superconducting gap energy.

The competition between CT and CAR has been analysed before ${ }^{40,41,47}$ and also different ways of separating the contributions experimentally have been considered $^{36,37,51,93}$. However, in this review article we analyse the intriguing effects of e-e interactions within the QWs for the NSN junction case. It is worth emphasizing here that if such NSN junctions are made out of 1-D systems like GaAs QWs or carbon nanotubes, then the effect of e-e interactions can influence the transport properties and low energy dynamics of such systems significantly.

The Landauer-Buttiker conductance matrix for the NSN case can be written, in the subgapped regime where $k_{B} T, e V_{i} \ll \Delta$, as ${ }^{40}$

$$
\left[\begin{array}{c}
I_{1} \\
I_{2}
\end{array}\right]=\left[\begin{array}{cc}
G_{A}+G_{C A}+G_{C T} & G_{C A}-G_{C T} \\
G_{C A}-G_{C T} & G_{A}+G_{C A}+G_{C T}
\end{array}\right]\left[\begin{array}{c}
V_{1} \\
V_{2}
\end{array}\right],
$$

The conductances here are related to the elements of the $\mathbb{S}$-matrix: $G_{A} \propto\left|r_{A}\right|^{2}$, $G_{C T} \propto|t|^{2}$ and $G_{C A} \propto\left|t_{A}\right|^{2} . G_{A}$ is the conductance due to the local AR that occurs at a single NS junction, whereas $G_{C T}$ and $G_{C A}$ are the conductance due to the elastic CT and CAR processes respectively, both of which involve transmissions between two QWs and give contributions with opposite signs to the sub-gap conductance between the two wires, $G_{C A}-G_{C T}$. The opposite signs in $G_{C A}-G_{C T}$ arise due to the electron and hole carriers with opposite charge.

\subsection{WIRG study of superconducting junctions}

We study the effects of inter-electron interactions on the $\mathbb{S}$-matrix using the renormalization group (RG) method introduced in Sec. 2 following Ref. 26, and the generalizations to multiple QWs in Refs. [27, 71]. The basic idea of the method is as follows. The presence of back-scattering (reflection) induces Friedel oscillations in the density of non-interacting electrons. Within a mean field picture for the weakly interacting electron gas, the electron not only scatters off the potential barrier but also scatters off these density oscillations with an amplitude proportional to the interaction strength. Hence by calculating the total reflection amplitude due to 
scattering from the scalar scatterer and also from the Friedel oscillations created by the scatterer in the presence of the e-e inside the QW, we can include the effect of e-e interaction in calculating transport. The above mentioned idea can now be generalized to the case where there is, besides non-zero reflection, also non-zero AR due to the presence of the superconducting boundary at the junction.

To derive the RG equations in the presence of Andreev processes, we here follow a similar procedure as described in Sec. 2. The fermion fields on each wire can be written as,

$$
\psi_{i s}(x)=\Psi_{I i s}(x) e^{i k_{F} x}+\Psi_{O i s}(x) e^{-i k_{F} x},
$$

where $i$ is the wire index, $s$ is the spin index which can be $\uparrow, \downarrow$ and $I, O$ stands for outgoing or incoming fields. Note that $\Psi_{I}(x)\left(\Psi_{O}(x)\right)$ are slowly varying fields on the scale of $k_{F}^{-1}$ and contain the annihilation operators as well as the slowly varying wave-functions. For a momentum in the vicinity of $k_{F}$, the incoming and outgoing fields (with the incoming field on the $i^{\text {th }}$ wire) can be Fourier expanded in terms of the scattering states and the electron field can be written as

$$
\begin{aligned}
\Psi_{i s}(x) & =\int d k\left[b_{k s} e^{i\left(k+k_{F}\right) x}+d_{k s}^{\dagger} e^{i\left(-k+k_{F}\right) x}\right. \\
& +r b_{k s} e^{-i\left(k+k_{F}\right) x}+r^{\star} d_{k s}^{\dagger} e^{-i\left(-k+k_{F}\right) x} \\
& \left.+r_{A} d_{k s} e^{-i\left(-k+k_{F}\right) x}+r_{A}^{\star} b_{k s}^{\dagger} e^{-i\left(k+k_{F}\right) x}\right] \\
\Psi_{(j \neq i) s}(x) & =\int d k\left[t b_{k s} e^{i\left(k+k_{F}\right) x}+t d_{k s}^{\dagger} e^{i\left(-k+k_{F}\right) x}\right. \\
& \left.+t_{A} d_{k s} e^{-i\left(-k+k_{F}\right) x}+t_{A}^{\star} b_{k s}^{\dagger} e^{-i\left(k+k_{F}\right) x}\right],
\end{aligned}
$$

where $b_{k s}$ is the electron destruction operator and $d_{k s}$ is the hole destruction operator. Note that we choose to quantise the fermions in the basis of the space of solutions of the Dirac equation i.e. around the linear spectrum, in the presence of a potential which allows for normal as well as Andreev scattering. We have also allowed for both incident electrons and holes. We find that (dropping a constant background density),

$$
\begin{aligned}
\left\langle\rho_{i s}(x)\right\rangle=\left\langle\Psi_{i s}^{\dagger} \Psi_{i s}\right\rangle= & \frac{i}{4 \pi x}\left[\left(r^{\star} e^{2 i k_{F} x}-r e^{-2 i k_{F} x}\right)\right. \\
& \left.+\left(r e^{2 i k_{F} x}-r^{\star} e^{-2 i k_{F} x}\right)\right]
\end{aligned}
$$

where the two terms corresponds to the density for electrons and holes respectively. Here we have also used the fact that due to the proximity of the superconductor, the amplitude to create (destroy) a spin $s$ electron and destroy (create) a spin $s$ hole is non-zero - i.e., the Boguliobov amplitudes $\left\langle d_{k-s}^{\dagger} b_{k s}^{\dagger}\right\rangle=1=\left\langle b_{k s} d_{k-s}\right\rangle$, besides the normal amplitudes $\left\langle d_{k s}^{\dagger} d_{k s}\right\rangle=\left\langle b_{k s} b_{k s}^{\dagger}\right\rangle=1$. (This is of course true only close to the superconductor i.e. of the order of the phase coherence length of the superconductor. We have checked that this gives the same result as solving the Boguliobov-de Gennes equation as done in Ref. [58, 59]). Hence, besides the density, 
the expectation values for the pair amplitudes $\left\langle\Psi_{i s}^{\dagger} \Psi_{i s}^{\dagger}\right\rangle$ and its complex conjugate $\left\langle\Psi_{i s} \Psi_{i s}\right\rangle$ are also non-zero and are given by (dropping the wire index)

$$
\begin{aligned}
\left\langle\psi_{O \uparrow}^{\dagger} \psi_{I \downarrow}^{\dagger}\right\rangle & =-\left\langle\psi_{O \downarrow}^{\dagger} \psi_{I \uparrow}^{\dagger}\right\rangle=\frac{-i r_{A}}{4 \pi x} \\
\text { and } \quad\left\langle\psi_{O \uparrow} \psi_{I \downarrow}\right\rangle & =-\left\langle\psi_{O \downarrow} \psi_{I \uparrow}\right\rangle=\frac{-i r_{A}^{\star}}{4 \pi x} .
\end{aligned}
$$

So, we see that the Boguliobov amplitudes also fall off as $1 / x$ just like the normal density amplitudes which is $1 / x^{\alpha}$ for strong e-e interaction inside the $\mathrm{QW}$ and using bosonization ${ }^{63}$.

We now allow for short-range density-density interactions between the fermions

$$
\mathcal{H}_{\text {int }}=\frac{1}{2} \int d x d y\left(\sum_{s=\uparrow, \downarrow} \rho_{s}\right) V(x-y)\left(\sum_{s=\uparrow, \downarrow} \rho_{s}\right),
$$

to obtain the standard four-fermion interaction Hamiltonian for the spin-full fermions as

$$
\begin{aligned}
\mathcal{H}_{\text {int }} & =\int d x\left[g _ { 1 } \left(\Psi_{I \uparrow}^{\dagger} \Psi_{O \uparrow}^{\dagger} \Psi_{I \uparrow} \Psi_{O \uparrow}+\Psi_{I \downarrow}^{\dagger} \Psi_{O \downarrow}^{\dagger} \Psi_{I \downarrow} \Psi_{O \downarrow}\right.\right. \\
& \left.+\Psi_{I \uparrow}^{\dagger} \Psi_{O \downarrow}^{\dagger} \Psi_{I \downarrow} \Psi_{O \uparrow}+\Psi_{I \downarrow}^{\dagger} \Psi_{O \uparrow}^{\dagger} \Psi_{I \uparrow} \Psi_{O \downarrow}\right) \\
& +g_{2}\left(\Psi_{I \uparrow}^{\dagger} \Psi_{O \uparrow}^{\dagger} \Psi_{O \uparrow} \Psi_{I \uparrow}+\Psi_{I \downarrow}^{\dagger} \Psi_{O \downarrow}^{\dagger} \Psi_{O \downarrow} \Psi_{I \downarrow}\right. \\
& \left.\left.+\Psi_{I \uparrow}^{\dagger} \Psi_{O \downarrow}^{\dagger} \Psi_{O \downarrow} \Psi_{I \uparrow}+\Psi_{I \downarrow}^{\dagger} \Psi_{O \uparrow}^{\dagger} \Psi_{O \uparrow} \Psi_{I \downarrow}\right)\right],
\end{aligned}
$$

where $g_{1}$ and $g_{2}$ are the running coupling constants defined in Sec. 2 (Eq. 23 and Eq. 24).

Now if we perform Hartree-Fock (HF) decomposition of the four-fermion interaction Hamiltonian (Eq. 32) allowing only particle number conserving amplitudes, then we get

$$
\begin{aligned}
\mathcal{H}_{\mathrm{int}}^{N} & =\int d x\left[g _ { 1 } \left[\left(\Psi_{I \uparrow}^{\dagger} \Psi_{O \uparrow}+\Psi_{I \downarrow}^{\dagger} \Psi_{O \downarrow}\right)+\left(\left\langle\Psi_{O \uparrow}^{\dagger} \Psi_{I \uparrow}\right\rangle+\left\langle\Psi_{O \downarrow}^{\dagger} \Psi_{I \downarrow}\right\rangle\right)\right.\right. \\
& \left.+\left(\Psi_{O \uparrow}^{\dagger} \Psi_{I \uparrow}+\Psi_{O \downarrow}^{\dagger} \Psi_{I \downarrow}\right)\left(\left\langle\Psi_{I \uparrow}^{\dagger} \Psi_{O \uparrow}\right\rangle+\left\langle\Psi_{I \downarrow}^{\dagger} \Psi_{O \downarrow}\right\rangle\right)\right] \\
& -g_{2}\left(\Psi_{I \uparrow}^{\dagger} \Psi_{O \uparrow}\left\langle\Psi_{O \uparrow}^{\dagger} \Psi_{I \uparrow}\right\rangle+\Psi_{O \uparrow}^{\dagger} \Psi_{I \uparrow}\left\langle\Psi_{I \uparrow}^{\dagger} \Psi_{O \uparrow}\right\rangle\right. \\
& \left.\left.+\Psi_{I \downarrow}^{\dagger} \Psi_{O \downarrow}\left\langle\Psi_{O \downarrow}^{\dagger} \Psi_{I \downarrow}\right\rangle+\Psi_{O \downarrow}^{\dagger} \Psi_{I \downarrow}\left\langle\Psi_{I \downarrow}^{\dagger} \Psi_{O \downarrow}\right\rangle\right)\right],
\end{aligned}
$$

Using the expectation values for the fermion operators given in Eq. 29, the effective interaction Hamiltonian (normal) can be derived in the following form on 
each half wire,

$$
\begin{aligned}
\mathcal{H}_{\mathrm{int}}^{N} & =\frac{-i\left(g_{2}-2 g_{1}\right)}{4 \pi} \int_{0}^{\infty} \frac{d x}{x}\left[r^{\star}\left(\Psi_{I \uparrow}^{\dagger} \Psi_{O \uparrow}+\Psi_{I \downarrow}^{\dagger} \Psi_{O \downarrow}\right)\right. \\
& \left.-r\left(\Psi_{O \uparrow}^{\dagger} \Psi_{I \uparrow}+\Psi_{O \downarrow}^{\dagger} \Psi_{I \downarrow}\right)\right] .
\end{aligned}
$$

(where we have assumed spin-symmetry i.e. $r_{\uparrow}=r_{\downarrow}=r$ ). Eq. 34 has been derived earlier in Ref. 27.

On the other hand, if we perform the HF deconposition of the four fermion terms allowing for particle non-conserving terms (pairing amplitude), then we obtain

$$
\begin{aligned}
\mathcal{H}_{\text {int }}^{A} & =\int d x\left[g _ { 1 } \left(\left\langle\Psi_{O \downarrow}^{\dagger} \Psi_{I \uparrow}^{\dagger}\right\rangle \Psi_{O \uparrow} \Psi_{I \downarrow}+\Psi_{O \downarrow}^{\dagger} \Psi_{I \uparrow}^{\dagger}\left\langle\Psi_{O \uparrow} \Psi_{I \downarrow}\right\rangle\right.\right. \\
& \left.+\left\langle\Psi_{O \uparrow}^{\dagger} \Psi_{I \downarrow}^{\dagger}\right\rangle \Psi_{O \downarrow} \Psi_{I \uparrow}+\Psi_{O \uparrow}^{\dagger} \Psi_{I \downarrow}^{\dagger}\left\langle\Psi_{O \downarrow} \Psi_{I \uparrow}\right\rangle\right) \\
& -g_{2}\left(\left\langle\Psi_{O \downarrow}^{\dagger} \Psi_{I \uparrow}^{\dagger}\right\rangle \Psi_{O \downarrow} \Psi_{I \uparrow}+\Psi_{O \downarrow}^{\dagger} \Psi_{I \uparrow}^{\dagger}\left\langle\Psi_{O \downarrow} \Psi_{I \uparrow}\right\rangle\right. \\
& \left.\left.+\left\langle\Psi_{O \uparrow}^{\dagger} \Psi_{I \downarrow}^{\dagger}\right\rangle \Psi_{O \uparrow} \Psi_{I \downarrow}+\Psi_{O \uparrow}^{\dagger} \Psi_{I \downarrow}^{\dagger}\left\langle\Psi_{O \uparrow} \Psi_{I \downarrow}\right\rangle\right)\right],
\end{aligned}
$$

Using Eq. 30 in $\mathcal{H}_{\text {int }}^{A}$, we get the (Andreev) Hamiltonian which can be written as

$$
\begin{aligned}
\mathcal{H}_{\text {int }}^{A}= & \frac{-i\left(g_{1}+g_{2}\right)}{4 \pi} \int_{0}^{\infty} \frac{d x}{x}\left[-r_{A}^{\star}\left(\Psi_{I \uparrow}^{\dagger} \Psi_{O \downarrow}^{\dagger}+\right.\right. \\
& \left.\left.\Psi_{O \uparrow}^{\dagger} \Psi_{I \downarrow}^{\dagger}\right)+r_{A}\left(\Psi_{O \downarrow} \Psi_{I \uparrow}+\Psi_{I \downarrow} \Psi_{O \uparrow}\right)\right] .
\end{aligned}
$$

Note that although Eq. 36 appears to be charge non-conserving, charge conservation is taken care of by the $2 e$ charge that flows into the superconductor every time there is an Andreev process taking place.

The amplitude to go from an incoming electron wave to an outgoing electron wave under $e^{-i \mathcal{H}_{\text {int }}^{N} t}$ (for electrons with spin) was derived in Ref. 27 and is given by

$$
\frac{-\alpha r_{s}}{2} \ln (k d),
$$

where $\alpha=\left(g_{2}-2 g_{1}\right) / 2 \pi \hbar v_{F}$ and $d$ was a short distance cut-off. Analogously, the amplitude to go from an incoming electron $e_{\text {in }}$ wave to an outgoing hole $h_{\text {out }}$ wave under $e^{-i \mathcal{H}_{\text {int }}^{A} t}$ is given by

$$
\begin{aligned}
& e^{-i \mathcal{H}_{\text {int }}^{A} t}\left|\mathrm{e}_{\text {in }}, s, k\right\rangle, \\
& \quad=-i \int \frac{d k^{\prime}}{2 \pi}\left[\left|\mathrm{h}_{\text {out }}, s^{\prime}, k^{\prime}\right\rangle\left\langle\mathrm{h}_{\text {out }}, s^{\prime}, k^{\prime}\left|\mathcal{H}_{\text {int }}^{A}\right| \mathrm{e}_{\text {in }}, s, k\right\rangle\right], \\
& \quad=\frac{-i\left(g_{1}+g_{2}\right) r_{A}}{4 \pi \hbar v_{F}} \int \frac{d x}{x} e^{-2 i k x}\left|\mathrm{~h}_{\text {out }}, s^{\prime}, k^{\prime}\right\rangle .
\end{aligned}
$$


where $s \neq s^{\prime}$. Hence, the amplitude for an incoming electron to be scattered to an outgoing hole by the Andreev process is given by

$$
\frac{\alpha^{\prime} r_{A}}{2} \ln (k d)
$$

where $\alpha^{\prime}=\left(g_{1}+g_{2}\right) / 2 \pi \hbar v_{F}$. Note also that $\alpha$ and $\alpha^{\prime}$ are themselves momentum dependent, since the $g_{i}$ 's are momentum dependent i.e. running coupling constants. For the spinful case, the amplitude for an incoming electron to go to an outgoing electron on the same QW is governed by the interaction parameter $\alpha=\left(g_{2}-\right.$ $\left.2 g_{1}\right) / 2 \pi \hbar v_{F}$ which has the possibility of chaging sign under RG evolution, because of the relative sign between $g_{1}$ and $g_{2}$. On the other hand, $\alpha^{\prime}=\left(g_{2}+g_{1}\right) / 2 \pi \hbar v_{F}$ can never change its sign as they are of the same sign.

\subsubsection{NS Junction}

In is shown before that the amplitudes in Eqs. 37 and 39 are corrections to the reflections of electrons from Friedel oscillations (as an electron) and from the pair potential (as a hole) respectively. We can combine them with the bare $\mathbb{S}$-matrix at the junction to find the corrections to the amplitudes of the bare $\mathbb{S}$-matrix. For an NS junction, there is only one wire coupled to the superconductor and the $\mathbb{S}$-matrix is just $2 \times 2$ for each value of the spin and is given by

$$
\mathbb{S}=\left[\begin{array}{cc}
r & r_{A} \\
r_{A} & r
\end{array}\right]
$$

Here $r$ is the bare normal refelction amplitude and $r_{A}$ is the bare AR amplitude from the NS interface. So we only need to compute the corrections to $r$ and $r_{A}$ in this case.

We find that there are five processes which contribute to the amplitude $r_{A}$ to first order in the repulsive e-e interaction parameter. The following diagrams are illustrated in Fig. 5. Adding all the contributions, we obtain the renormalized AR amplitude $r_{A}$ that takes an incoming electron to an outgoing hole and is given by

$$
\delta r_{A}=\frac{\alpha^{\prime}}{2}\left[r_{A}-r_{A}^{\star}\left(r^{2}+r_{A}^{2}\right)\right] \ln (k d)+\alpha|r|^{2} r_{A} \ln (k d),
$$

which is in agreement with Ref. [58, 59]. On the other hand, for an incoming electron reflected back as an electron, we find the small correction in the amplitude $\delta r$ given by 26,27

$$
\delta r=-\alpha^{\prime}\left|r_{A}\right|^{2} r \ln (k d)+\frac{\alpha}{2}\left[r_{A}^{2} r^{\star}-r\left(1-|r|^{2}\right)\right] \ln (k d),
$$

We replace $-\ln (k d)$ by $d l$ using the "poor man's scaling" approach ${ }^{69}$ to obtain the $\mathrm{RG}$ equation for $r_{A}$ as

$$
\frac{d r_{A}}{d l}=-\frac{\alpha^{\prime}}{2}\left[r_{A}-r_{A}^{\star}\left(r^{2}+r_{A}^{2}\right)\right]-\alpha|r|^{2} r_{A}
$$




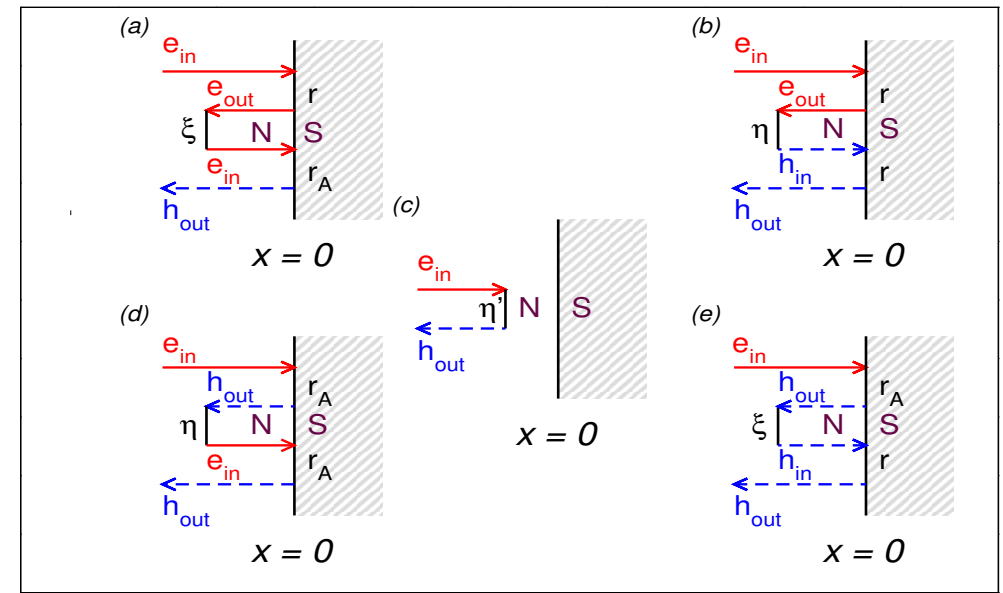

Fig. 5. (Color online) The processes that contribute to the amplitude for an incoming electron to transform to an outgoing hole. Note that all the processes shown here are to first order in the interaction parameters since they only involve a single scattering from a Friedel oscillation or the pair potential. Process $(c)$ involves scattering from a pair potential before the electron reaches the junction. The remaining processes involve two reflections from the junction and a scattering from the Friedel oscillation or the pair potential. In the diagrams, $\xi=\frac{1}{2} \alpha r^{\star} \ln (k d)$, $\eta=-\frac{1}{2} \alpha^{\prime} r_{A}^{\star} \ln (k d)$ and $\eta^{\prime}=\frac{1}{2} \alpha^{\prime} r_{A} \ln (k d)$. Figure adapted from Ref. 75.

Using the unitarity of the $\mathbb{S}$-matrix $\left(\left|r_{A}\right|^{2}+\left|r^{2}\right|=1\right.$ and $\left.r_{A}^{\star} r+r_{A} r^{\star}=0\right)$, we can simplify the RHS of the Eq. 43 to obtain

$$
\frac{d r_{A}}{d l}=-\left(\alpha+\alpha^{\prime}\right) r_{A}\left(1-\left|r_{A}\right|^{2}\right),
$$

Note that the combination $\alpha+\alpha^{\prime}=\left(2 g_{2}-g_{1}\right) / 2 \pi \hbar v_{F}$ which appears in the RG equation (Eq. 44) does not flow under RG. The latter can be seen from Eqs. 23 and 24 which shows that $\left(2 g_{2}-g_{1}\right) / 2 \pi \hbar v_{F}=\left(2 V(0)-V\left(2 k_{F}\right)\right) / 2 \pi \hbar v_{F}$. The following observation implies that $r$ and $r_{A}$ either monotonically increase or decrease as a power law depending on the sign of $\alpha+\alpha^{\prime}$. From Eq. 44, we also observe that $\left|r_{A}\right|=0$ and $\left|r_{A}\right|=1$ correspond to the insulating and the Andreev fixed points of the NS junction respectively. One can easily see from the RG equations that $\left|r_{A}\right|=0$ is a stable fixed point and $\left|r_{A}\right|=1$ is an unstable fixed point. Due to a small perturbation around the unstable $\left|r_{A}\right|=1$ fixed point, the system always flows towards the stable $|r|=0$ fixed point in which the QW is completely disconneced from the superconducting junction.

\subsubsection{NSN Junction}

In this subsection, we consider a NSN junction. Here in addition to the two reflection channels, we also have two channels for transmission - the direct transmission of an electron to an electron through CT process and the transmission of an incoming 
electron to a outgoing hole via CAR. These two processes are depicted in Fig. 6 . The $\mathbb{S}$-matrix at the junction is $8 \times 8$ in this case and is given in Eq. 25. The number of scattering processes from the Friedel oscillations and pair potentials that contribute to the renormalization of the scattering amplitudes in this case is thirty four, since we also need to include terms that transmit electrons or holes through the junction. For instance, for the renormalization of the AR term, besides the terms corresponding to the NS junction, we also have to include processes in which the incident electron from wire 1, goes through the junction to wire 2, Andreev reflects from the pair potential on wire 2 and then comes back through the junction to wire 1, as shown pictorially in Fig. 7(c).

Collecting all the nine processes that contribute to first order in $\alpha$ and $\alpha^{\prime}$ to the reflection amplitude, we find that

$$
\frac{d r}{d l}=-\left[\frac{\alpha}{2}\left[\left(t^{2}+r_{A}^{2}+t_{A}^{2}\right) r^{\star}-r\left(1-|r|^{2}\right)\right]-\alpha^{\prime}\left(r\left|r_{A}\right|^{2}+r_{A}^{\star} t_{A} t\right)\right],
$$

Similarly, adding up the contributions from the nine processes that contribute to $r_{A}$, we find that

$$
\frac{d r_{A}}{d l}=-\left[\alpha\left(|r|^{2} r_{A}+t t_{A} r^{\star}\right)+\frac{\alpha^{\prime}}{2}\left(r_{A}-\left(r^{2}+r_{A}^{2}+t^{2}+t_{A}^{2}\right) r_{A}^{\star}\right)\right],
$$

Moreover, for the NSN case, besides the reflection parameters, we also need to compute the renormalizations of the transmissions (CT and CAR) to first order in $\alpha$ and $\alpha^{\prime}$. The RG equations for $t$ and $t_{A}$ are also obtained by considering all possible processes that ultimately have one incoming electron and one outgoing electron (for $t$ ) and one incoming electron and one outgoing hole (for $t_{A}$ ) which are either reflected once from the Friedel potential or the pair potential. Finally they are found to be

$$
\begin{aligned}
\frac{d t}{d l} & =-\left[\alpha\left(|r|^{2} t+r^{\star} r_{A} t_{A}\right)-\alpha^{\prime}\left(\left|r_{A}\right|^{2} t+r r_{A}^{\star} t_{A}\right)\right], \\
\frac{d t_{A}}{d l} & =-\left[\alpha\left(r^{\star} r_{A} t+|r|^{2} t_{A}\right)-\alpha^{\prime}\left(r t r_{A}^{\star}+\left|r_{A}\right|^{2} t_{A}\right)\right] .
\end{aligned}
$$
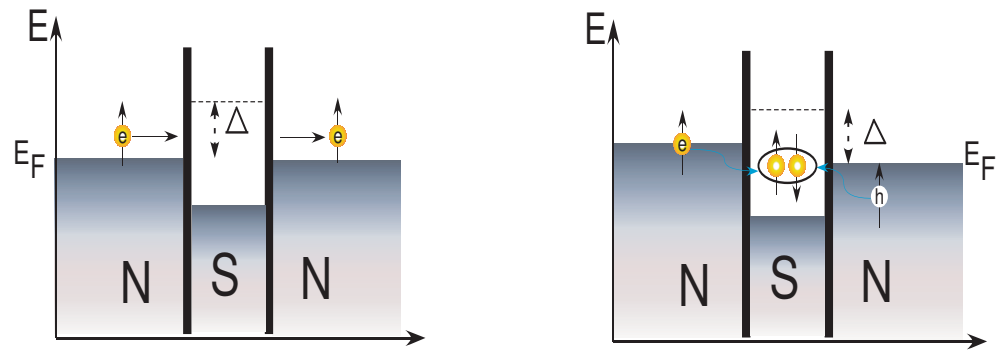

Fig. 6. (Color online) Electron CT with bare amplitude $t$ is shown in the left figure and CAR with bare amplitude $t_{A}$ is shown in the right diagram. Figure adapted from Ref. 75 . 


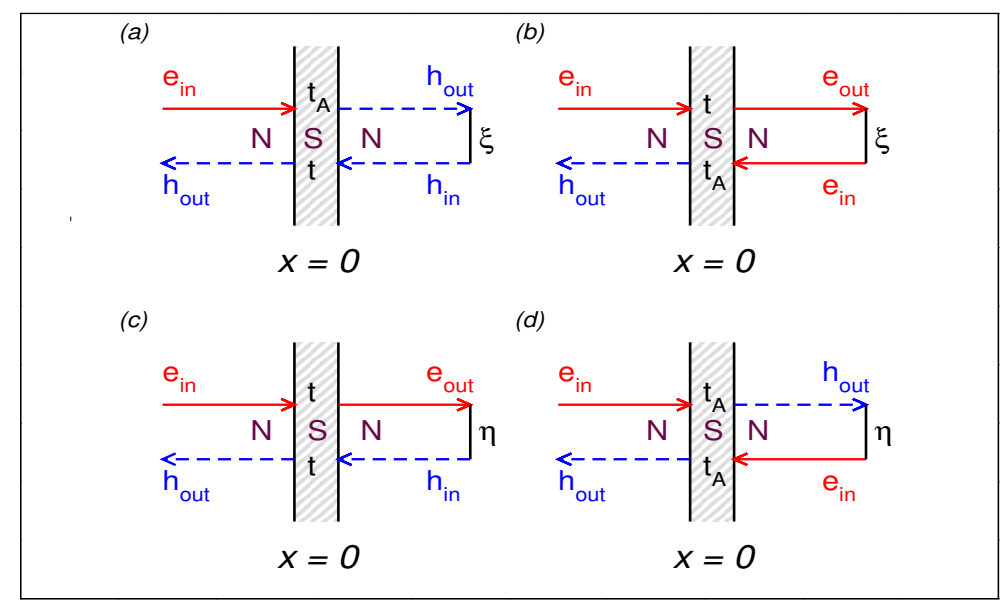

Fig. 7. The extra processes that contribute to the amplitude for an incoming electron to transform to an outgoing hole on the same wire, due to the second wire. In processes (a) and (b) the incident electron from the first wire are transmitted to the second wire and reflected by the Friedel oscillation whereas (c) and (d) are transmitted to the second wire and reflected by the pair potential. Figure adapted from Ref. 75 .

It has been emphasized in Sec. 2 that for the normal junction case the RG equations can be expressed in a compact from (Eq. 20) ${ }^{27}$. Similarly we can express the RG equations for the superconducting junction case also in a compact matrix form as given below,

$$
\frac{d \mathbb{S}}{d l}=\tilde{F}-\mathbb{S} \tilde{F}^{\dagger} \mathbb{S}
$$

where the matrix $\mathbb{S}$ is given in Eq. 25 and $\tilde{F}$ depends on the interaction parameters $\alpha=\left(g_{2}-2 g_{1}\right) / 2 \pi \hbar v_{F}$ and $\alpha^{\prime}=\left(g_{1}+g_{2}\right) / 2 \pi \hbar v_{F}$ in the QW. $\tilde{F}$ is non-diagonal matrix (unlike the case in Ref. 27) and is given by

$$
\tilde{F}=\left[\begin{array}{cccc}
\frac{\alpha r}{2} & 0 & \frac{-\alpha^{\prime} r_{A}}{2} & 0 \\
0 & \frac{\alpha r}{2} & 0 & \frac{-\alpha^{\prime} r_{A}}{2} \\
\frac{-\alpha^{\prime} r_{A}}{2} & 0 & \frac{\alpha r}{2} & 0 \\
0 & \frac{-\alpha^{\prime} r_{A}}{2} & 0 & \frac{\alpha r}{2}
\end{array}\right] .
$$

It is now easy to check that all the RG equations can be reproduced from the matrix equation. The matrix form also makes the generalization to $N$ wires case notationally simple and makes the search for various fixed point much easier. But note that these equations have to be augmented by Eqs. 23 and 24 to get the full set of RG equations. 
Let us now look at some of the fixed points of the $\mathbb{S}$-matrix. Clearly, the fixed points occur when $\tilde{F}-\mathbb{S} \tilde{F}^{\dagger} \mathbb{S}=0$ or when $\tilde{F} \mathbb{S}^{\dagger}$ is hermitian. There are several possibilities and we list below some important of them.

Case I: Any one of the four amplitudes is non-zero

(a) $t=1, r=r_{A}=t_{A}=0$, fully transmitting fixed point (TFP)

(b) $r=1, r_{A}=t_{A}=t=0$ fully reflecting fixed point (RFP)

(c) $r_{A}=1, r=t=t_{A}=0$, fully Andreev reflecting fixed point (AFP)

(d) $t_{A}=1, r=t=r_{A}=0$, fully crossed Andreev reflecting fixed point (CAFP)

Case II: Any two amplitudes are non-zero

When both $r$ and $r_{A}$ are zero, the RHS of the RG equations identically vanishes as both the Friedel oscillation amplitude as well as the pair potential amplitude in the wire become zero. Hence any value of $t$ and $t_{A}$ remains unrenormalized under RG.

Case III: Any three of them are non-zero

We did not find any fixed point of this kind.

Case IV: All four of them are non-zero

Here, we get a fixed point when $r_{1}=r_{2}=t=t_{A}=1 / 2$ and $r_{A 1}=r_{A 2}=-1 / 2$. The latter is the most symmetric $\mathbb{S}$-matrix possible for the NSN case. Since it is a symmetry-dictated fixed point with intermediate transmission and reflection, we shall refer to it as symmetric fixed point (SFP).

\subsubsection{FS, FSF and FSN Junctions}

We can also consider junctions where one or more of the wires are spin-polarised, with Fermi distributions for the spin up and down electrons being different. As long as at least one of the wires is ferromagnetic, the spin up-spin down symmetry of the system is broken. This implies that we can no longer impose $\mathbb{S}_{\uparrow}=\mathbb{S}_{\downarrow}$ on the $\mathbb{S}$-matrix parametrising the scattering at the junction as we had before in Eq. 25. We now need to choose an $\mathbb{S}$-matrix with indices $\uparrow$ and $\downarrow$ denoting the spin. Experimental set-up based on Ferromagnet-Superconductor (FS) hybrid structures has been investigated in the recent past ${ }^{37,113}$. For the Ferromagnet-Superconductor-Normal (FSN) case (and the Ferromagnet-Superconductor-Ferromagnet (FSF) case where the ferromagnets on the two sides are not identically polarized) the wire index symmetry is also broken due to the spin assymetry in the two wires. Hence, the $\mathbb{S}$-matrix chosen must also break the wire-index symmetry. Note that for the ferromagnetic wire, the amplitude to destroy a spin $s$ electron and create a spin $s$ hole cannot be non-zero, even in the proximity of the superconductor. The Boguliobov amplitudes $\left\langle d_{i k-s}^{\dagger} b_{i k s}^{\dagger}\right\rangle$ and $\left\langle b_{i k s} d_{i k-s}\right\rangle$ decay exponentially fast (with a length scale set by the ferro-anti-ferro gap) in the ferromagnetic wire. So, in our $\mathbb{S}$-matrix, $r_{A}$ is zero and there is no pair potential due to the proximity effect in ferromagnetic wire. Also as mentioned earlier, we must keep in mind that the influence of the bulk ferromagnet on the spectrum of the superconductor and on the QW have to be 
negligibly small. This will be true only if the superconductor is large enough. Hence, for such junctions, the renormalization of the $\mathbb{S}$-matrix sets in only scattering due to the Friedel oscillations. Also note that in these wires, since the bulk does not have both the spin species, $g_{1}$ and $g_{2}$ do not get renormalized i.e. they are not running coupling constant anymore. All the cases mentioned above will therefore involve the full $4 N \times 4 N \mathbb{S}$-matrix since there is no reduction in number of independent elements of the $\mathbb{S}$-matrix which can occur when symmetries are imposed.

\subsubsection{Three-Wire-Junction-A Beam Splitter}

In this subsection, we consider the standard beam splitter geometry comprising of a superconductor at the junction of three quantum wires. In this case, we show that there is a fixed point that is analogous to the Andreev fixed point of the NS junction. The $\mathbb{S}$-matrix representing this fixed point is symmetric under all possible permutations of the three QWs and allows for the maximum Andreev transmission (in all channels simultaneously within unitarity constraints). The $\mathbb{S}$-matrix is given by $r_{A}=-1 / 3$ and $t_{A}=t_{A}^{\prime}=2 / 3$ with $r=t=t^{\prime}=0$. We refer to this fixed point as the Andreev-Griffith's fixed point (AGFP) which is also an intermediate fixed point with non zero scattering amplitudes. Very recently, similar Cooper pair beam splitter geometry has been realized experimentally in the context of two-particle correlations of the shot noise of the split electrons via CAR process $53,54,57,114$. In literature the Griffith's fixed point represents the most symmetric $\mathbb{S}$-matrix for a normal three wire junction. It is given by $r=-1 / 3$ and $t=2 / 3$ where $r$ is the reflection within each wire and $t$ is the transmission from one wire to the other. The boundary condition for the three wire junction corresponding to the above mentioned $\mathbb{S}$-matrix was obtained by Griffith ${ }^{92}$ hence we refer to it as the Griffith's fixed point (GFP).

For an analytic treatment of this case, we consider a simplified situation where there is a complete symmetry between two of the wires, say 1 and 2 , and the $\mathbb{S}$ matrix is real. In addition, the elements of the $\mathbb{S}$-matrix corresponding to normal transmission or reflection of an incident electron (hole) to a reflected or transmitted electron (hole) are set to zero so that only Andreev scattering processes participating in transport. Then the $\mathbb{S}$-matrix is given by

$$
\mathbb{S}=\left[\begin{array}{cccccc}
0 & 0 & 0 & r_{A} & t_{A} & t_{A}^{\prime} \\
0 & 0 & 0 & t_{A} & r_{A} & t_{A}^{\prime} \\
0 & 0 & 0 & t_{A}^{\prime} & t_{A}^{\prime} & r_{A}^{\prime} \\
r_{A} & t_{A} & t_{A}^{\prime} & 0 & 0 & 0 \\
t_{A} & r_{A} & t_{A}^{\prime} & 0 & 0 & 0 \\
t_{A}^{\prime} & t_{A}^{\prime} & r_{A}^{\prime} & 0 & 0 & 0
\end{array}\right],
$$


where, $r_{A}$ and $t_{A}$ and $t_{A}^{\prime}$ are real parameters which satisfy ${ }^{65}$

$$
\begin{aligned}
t_{A} & =1+r_{A}, \\
r_{A}^{\prime} & =-1-2 r_{A}, \\
t_{A}^{\prime} & =\sqrt{\left(-2 r_{A}\right)\left(1+r_{A}\right)}, \\
\text { and } \quad-1 & \leq r_{A} \leq 0
\end{aligned}
$$

by unitarity. Using Eq. 52, the simplified RG equation for the single parameter $r_{A}$ is given by

$$
\frac{d r_{A}}{d l}=\alpha^{\prime}\left[r_{A}\left(1+r_{A}\right)\left(1+3 r_{A}\right)\right]
$$

So, within the real parametrization we have two unstable fixed points, given by

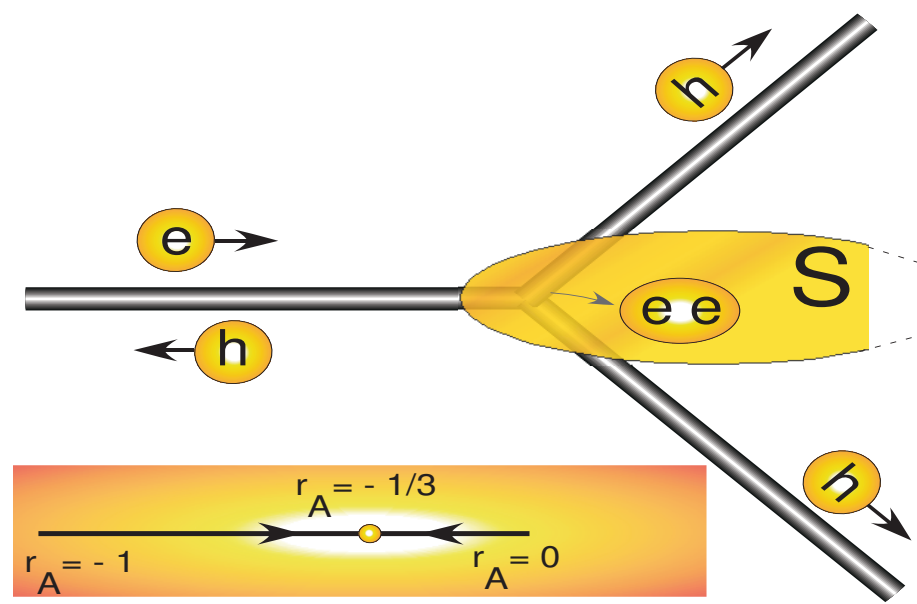

Fig. 8. (Color online) Schematic representation of the beam splitter geometry where a three-wire-junction is hooked to the stable fixed point, AGFP. An incident electron in one wire is either reflected back as a hole in the same wire (AR) or is transmitted as a hole (CAR) in another wire along with the addition of the two electrons into the superconductor forming a Cooper pair. The direction of RG flow from two unstable fixed points to the stable fixed point (AGFP) is also depicted on the bottom left side of the diagram. Figure adapted from Ref. 75.

$r_{A}=0$ and $r_{A}=-1$ and a stable fixed point given by $r_{A}=-1 / 3$. The $r_{A}=0$ fixed point corresponds to a situation where there is perfect CAR between wires 1 and 2 and wire 3 gets cut off from the remaining two wires (labelled by 1 and 2) and is in the perfect AR limit with the superconductor. The $r_{A}=-1$ fixed point corresponds to a situation where all the three wires are disconnected from each other and are in perfect AR limit individually with the superconductor. The third fixed point given by $r_{A}=-1 / 3$ corresponds to a perfect Andreev limit of the three wire junction where an incident electron is either Andreev-reflected into the same wire as a hole or is transmitted as a hole via CAR into another wire. This is 
essentially the AGFP. It is very interesting to note that the original Griffith's fixed point was a repulsive fixed point ${ }^{27,65}$ whereas the AGFP is an attractive fixed point. This can be understood as follows. In the current situation, there is no scattering from the Friedel oscillations as the junction is assumed to be reflection-less $(r=0)$, whereas there exists a proximity induced pair potential, which induces an effective attractive interaction between the electrons. Hence, the physics is very similar to the well-known LL physics, which says that for attractive interaction between the electrons, back-scattering is an irrelevant operator. Hence the stable fixed point here will be the one which will have maximal transmission between the wires. So, it is not surprising that the AGFP turns out to be a stable fixed point. Thus, for a reflectionless junction, we have found a stable fixed point with intermediate transmission and reflection analogus to the GFP in three wire junction ${ }^{27}$.

\subsection{Results of RG flows for the conductance}

In this subsection, we consider various physical cases regarding the RG fixed points and discuss the outcome of RG flows for the LB conductances in each case.

\subsubsection{NS Junction}

We start with the results for the NS junction, just to contrast with the results of the NSN junction in the next case. Here, we have only two parameters, $r$ and $r_{A}$. The conductance occurs only due to the AR amplitude, $r_{A}$ which obeys the RG equation given by Eq. 41. As mentioned earlier, there is no flow of the particular linear combination of the interaction parameters $2 g_{2}-g_{1}$ that occurs in the equation and hence the RG flow of the conductance is therefore monotonic. The conductance as a function of the length scale for different interaction parameters $V(0)$ and $V\left(2 k_{F}\right)$ is plotted in Fig. 9. Here $L$ simply denotes the length at which the RG is cut-off. So if we take very long wires $L_{W} \gg L_{T}$, then the cut-off is set by the temperature, and the plot shows the variation of the conductance as a function of $L_{T}$ starting from the high temperature limit, which here is the superconducting gap $\Delta$. We observe that as we lower the temperature, the Andreev subgapped conductance decreases monotonically with a power law set by the LL parameter and finally becomes zero when the QW is disconnected from the superconducting junction at $r=1$ stable fixed point. Also it was established in Ref. 59 that the power law scaling of conductance $\left(\left|r_{A}\right|^{2}\right)$ calculated from WIRG and bosonization were found to be in agreement with each other for the limiting cases of $\left|r_{A}\right|^{2} \cong 1$ and $\left|r_{A}\right|^{2} \cong 0$ (which are the only limits where bosonisation results are valid) provided effects due to electron-electron induced back-scattering in the wires is neglected. 


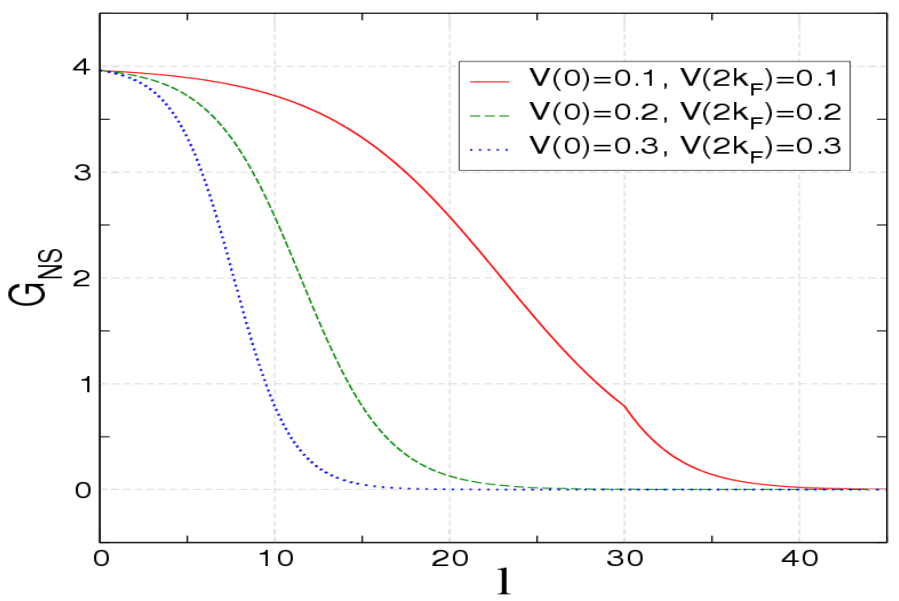

Fig. 9. (Color online) Conductance of the NS junction is plotted in units of $e^{2} / h$ as a function of the dimensionless parameter $l$ where $l=\ln (L / d)$ and $L$ is either $L_{T}=\hbar v_{F} / k_{B} T$ at zero bias or $L_{V}=\hbar v_{F} / \mathrm{eV}$ at zero temperature and $d$ is the short distance cut-off for the RG flow. The three curves correspond to three different values of $V(0)$ and $V\left(2 k_{F}\right)$. Figure adapted from Ref. 75 .

\subsubsection{Ballistic NSN Junction}

Here, we consider the case of a reflection-less ballistic junction between the superconductor and the two QWs, i.e. $r=0$. This implies that the renormalization of the S-matrix due to the Friedel oscillations is absent. The only renormalization of the $\mathbb{S}$-matrix elements occur due to reflections from the proximity effect induced pair potential inside the QW. Let us now consider various interesting cases:

(a) $r=0, r_{A}=0, t \neq 0, t_{A} \neq 0$.

In this case, since we have both $r=0$ and $r_{A}=0$, there is no RG flow of the transmissions and the conductance is frozen at the value that it had for the bare $\mathbb{S}$-matrix. The most interesting situation in this case arises when $t=t_{A}$. For this case, the probability for an incident electron in one wire, to transmit in the other wire as an electron due to $t$ or as hole due to $t_{A}$ is equal, leading to perfect cancellation of charge current.

(b) $r=0, t=0, r_{A} \neq 0, t_{A} \neq 0$.

For this case, one can easily check from the RG equations (Eqs. 45-48) that if we start our RG flow with the given parameters at high energies, then the value of $r, t$ remain stuck to the value zero under the RG flow. Hence, in this case the two parameter subspace $r_{A} \neq 0, t_{A} \neq 0$ remains secluded under the RG flow. The RG equation for $t_{A}$ is given by

$$
\frac{d t_{A}}{d l}=\alpha^{\prime} t_{A}\left(1-\left|t_{A}\right|^{2}\right)
$$


The above equation can be integrated to obtain an expression for CAR probability $\left(T_{A}=\left|t_{A}\right|^{2}\right)$,

$$
T_{A}(L)=\frac{T_{A}^{0}\left[\left[1+2 \alpha_{1} \ln \left(\frac{L}{d}\right)\right]^{\frac{3}{2}}\left(\frac{d}{L}\right)^{-\left(2 \alpha_{2}-\alpha_{1}\right)}\right]}{R_{A}^{0}+T_{A}^{0}\left[\left[1+2 \alpha_{1} \ln \left(\frac{L}{d}\right)\right]^{\frac{3}{2}}\left(\frac{d}{L}\right)^{-\left(2 \alpha_{2}-\alpha_{1}\right)}\right]} .
$$

where $T_{A}^{0}$ and $R_{A}^{0}$ are the CAR and AR probabilities respectively at the short distance cut-off, $L=d$. We notice that the RG equation and its solution are very similar to that for the single scatterer problem ${ }^{67}$ apart from a sign difference on the RHS of Eq. 55 and the dependance of the interaction parameter $\alpha^{\prime}$ on $g_{1}$ and $g_{2}$. This implies that even if we start with a small crossed Andreev transmission across the junction, the RG flow will take us towards the limit of perfect transmission. This is in sharp contrast to the normal transmission across a single scatterer. For the single barrier problem, the equation for the RG flow of $t$ was by

$$
\frac{d t}{d l}=-\alpha t\left(1-|t|^{2}\right) .
$$

Hence, $t=0$ is the stable fixed point in this case. But if the e-e interactions had been attractive, then the sign on the RHS would have been positive and $t=1$ would have been the stable fixed point. Thus, the RG flow of $t_{A}$ for the case when $r_{A} \neq 0, t=r=0$, and repulsive interactions, is very similar to the RG flow for $t$ when $r \neq 0, t_{A}=r_{A}=0$ but with attractive interactions. In both cases transmission is relevant and $t=1$ and $t_{A}=1$ are the stable fixed points. On the other hand the RG flow of $t_{A}$ for the case of $r_{A} \neq 0, t=0, r=0$ and attractive e-e interaction $\left(V(0), V\left(2 k_{F}\right)<0\right)$ in the wire is very similar to the RG flow for $t$ for the case $r \neq 0, r_{A}=0, t_{A}=0$ and repulsive e-e interaction $\left(V(0), V\left(2 k_{F}\right)>0\right)$. In both cases transmission is irrelevant and $t=0$ and $t_{A}=0$ are the stable fixed points. At an intuitive level, one can perhaps say that even if we start with repulsive inter-electron interactions inside the QW, the proximity-induced pair potential leads to a net attractive interaction between the electrons, which is responsible for the counter-intuitive RG flow.

Also note that while solving the above RG equation for $t_{A}$, we have to take into account the RG flow of the interaction parameter $\left(\alpha^{\prime}\right)$ itself. This will lead to non-power law (non Luttinger) behavior for the conductance close to $\left|t_{A}\right| \simeq 0$ or $\left|t_{A}\right| \simeq 1$. It is worth pointing out that the non-power law part appearing in Eq. 55 is identical to Ref. 67, even though the interaction parameter for their case was proportional to $g_{2}-2 g_{1}$ and for our case it is $g_{2}+g_{1}$. But of course the latter will not lead to any non-monotonic behavior as $\alpha^{\prime}$ can not change sign under RG flow. So the stable fixed point for this case is the CAFP.

(c) $r=0, t_{A}=0, r_{A} \neq 0, t \neq 0$.

This case is identical to the case (b) discussed above except for the fact that we 
have to replace $t_{A}$ in the previous case by $t$. In this case also the two parameter subspace $r_{A} \neq 0, t \neq 0$ remains secluded under RG flow. The RG equation for $t$ is given by

$$
\frac{d t}{d l}=\alpha^{\prime} t\left(1-|t|^{2}\right) .
$$

Here also, $t=1$ remains the stable fixed point and $t=0$ is the unstable fixed point.

(d) $r=0, t \neq 0, r_{A} \neq 0, t_{A} \neq 0$.

In this case if we start from a symmetric situation, i.e. $t=t_{A}$, we can see from the RG equations in Eqs. 47 and 48 that both $t$ and $t_{A}$ have identical RG flows. So, the sub-gap conductance $G=G_{C A}-G_{C T}$ vanishes identically and remains zero through out the RG flow. Hence this $\mathbb{S}$-matrix can facilitate production of pure SC ${ }^{76}$ if we inject spin polarized electrons from one of the leads as the charge current gets completely filtered out at the junction. In Sec 5 we shall discuss the NSN and FSN junction from the spintronics application point of view in greater details.

\subsubsection{Ballistic FSF Junction}

Here, we consider the case where both the wires are spin polarized. In this case we can have two interesting possibilities, i.e. either both the wires have aligned spin polarization (ferromagnetic) or they have them anti-aligned (anti-ferromagnetic). In either case the AR amplitude is zero on each wire due to reasons explained earlier.

(a) When the two wires have their spins aligned, $t \neq 0$, but $t_{A}=0$ because for CAR to happen we need up (down) spin polarization in one wire and down (up) spin polarization on the other wire which is not possible in this case.

(b) When the two wires have their spins anti-aligned, then $t=0$, but $t_{A} \neq 0$ because the up (down) electron from one wire cannot tunnel without flipping its spin into the other wire. As there is no mechanism for flipping the spin of the electron at the junction, such processes are not allowed.

Hence these two cases can help in separating out and measuring amplitudes of the direct tunneling process (CT) and the CAR process experimentally ${ }^{37,93}$. Both these are examples of case II, since they have both $r=0$ and $r_{A}=0$. In this case, neither $t$ nor $t_{A}$ change under RG flow and hence conductance is not influenced by e-e interaction at all.

\subsubsection{Non-ballistic NSN Junction without AR on individual wires}

Here we consider an NSN junction with finite reflection in each wire and no AR in the individual wires. So the renormalization of the $\mathbb{S}$-matrix is purely due to the 


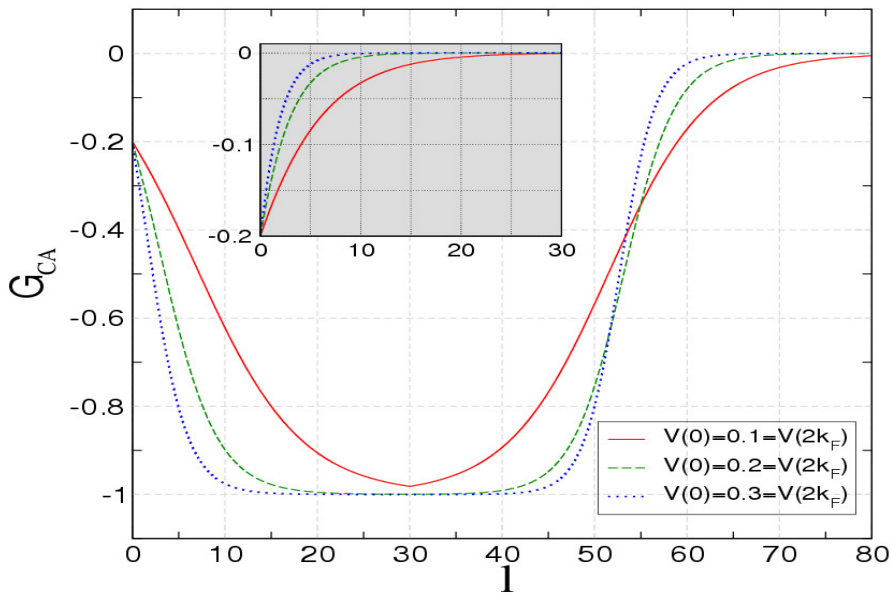

Fig. 10. (Color online) Conductance $G_{C A}$ of the NSN junction is plotted (when the two leads have anti-parallel spins) in units of $e^{2} / h$ as a function of the dimensionless parameter $l$ where $l=\ln (L / d)$ and $L$ is either $L_{T}=\hbar v_{F} / k_{B} T$ at zero bias or $L_{V}=\hbar v_{F} / e V$ at zero temperature and $d$ is the short distance cut-off for the RG flow. The three curves correspond to three different values of $V(0)$ and $V\left(2 k_{F}\right)$. The inset shows the behavior of the same conductance for fixed values of $\alpha$ i.e. for the spinless case. Figure adapted from Ref. 75 .

Friedel oscillations and there are no contributions originating from scattering due to the proximity induced pair potential. Below we discuss two cases :

(a) $r_{A}=0, t=0, r \neq 0, t_{A} \neq 0$.

The RG equations (Eqs. 45-48) predict that $r_{A}, t$ will remain zero under the $\mathrm{RG}$ flow and $r, t_{A}$ form a secluded sub-space. The RG equation for this case is given by

$$
\frac{d t_{A}}{d l}=-\alpha t_{A}\left(1-\left|t_{A}\right|^{2}\right) .
$$

Note the change in sign on the RHS with respect to the RG equation for $t_{A}$ (Eq. 54) for the ballistic case. This change in sign represents the fact that the ballistic case effectively represents a situation corresponding to attractive e-e interaction while the present case corresponds to a purely repulsive e-e interaction. In Fig. 10 we show the behavior of conductance $\left(G_{C A}\right)$ for this case. The conductance in the main graph shows a non-monotonic behavior due to the running coupling constant $\alpha$ in Eq. 58. To contrast, we also show in the inset, the behavior of the conductance for the spinless case when the renormalization of $\alpha$ in not taken into account. Thus, it is apparent from the plot that the non-monotonicity in the behavior of conductance manifests itself solely from the RG evolution of the running coupling constant $\alpha$. The inset and the main graph, both start from the same value of $t_{A}$. Even though this case is theoretically interesting to explore, its experimental realization may not be 
viable. This is because of the following reasons. Here we have $r_{A}=0$ on both wires, which can only happen if the wires are ferromagnetic. However, we also know that if the wires are ferromagnetic, there is no scaling of $\alpha$ parameter and hence there will be no interesting non-monotonic trend in the conductance. So it is hard to find a physical situation where $r_{A}=0$ and at the same time, there is renormalization of the interaction parameter $\alpha$. Lastly note that the conductance $G_{C A}$ is negative. The process responsible for the conductance, (i.e. CAR), converts an incoming electron to an outgoing hole or vice-versa, resulting in the negative sign.

(b) $r_{A}=0, t_{A}=0, r \neq 0, t \neq 0$.

This case is identical to the previous case with the replacement of $t_{A}$ by $t$. Fig. 11 shows the the CT conductance $G_{C T}$ as a function of the length scale. It shows a similar non-monotonic behavior with positive values for the conductance. The inset shows the behavior of $G_{C T}$ when the renormalization of $\alpha$ in not taken into account.

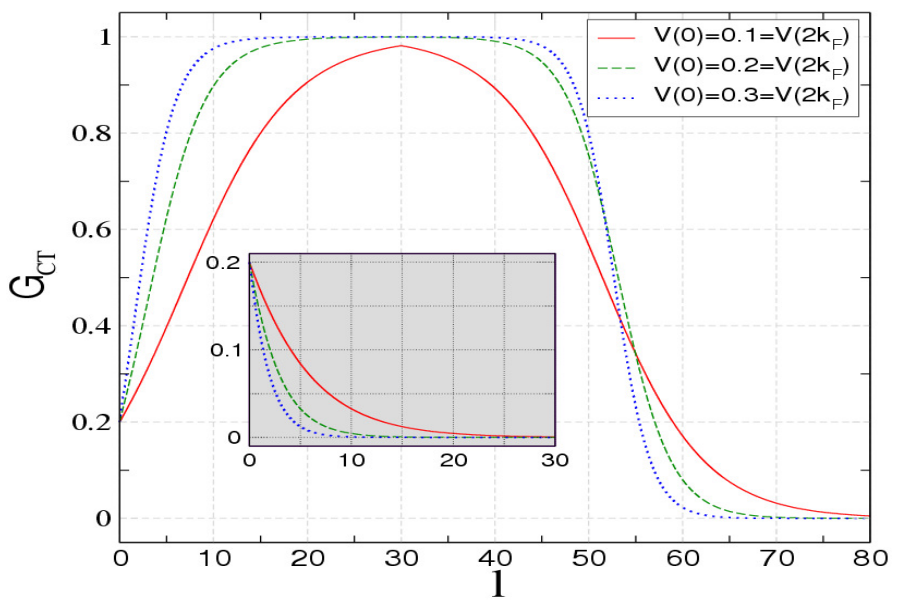

Fig. 11. (Color online) Conductance $G_{C T}$ of the NSN junction (when the two leads have parallel spins) in units of $e^{2} / h$ as a function of the dimensionless parameter $l$ where $l=\ln (L / d)$ and $L$ is either $L_{T}=\hbar v_{F} / k_{B} T$ at zero bias or $L_{V}=\hbar v_{F} / e V$ at zero temperature and $d$ is the short distance cut-off for the RG flow. The three curves correspond to three different values of $V(0)$ and $V\left(2 k_{F}\right)$. The inset shows the behavior of the same conductance for fixed values of $\alpha$ i.e. for the spinless case. Figure adapted from Ref. 75 .

\subsubsection{Non-ballistic NSN Junction with AR on individual wires}

For this case $r_{A} \neq 0, t_{A} \neq 0, r \neq 0, t \neq 0$ i.e. all the scattering amplitudes are non zero. This is the most interesting case, where both $r$ and $r_{A}$ are non-zero, and we 
get an interplay of the effects due to scattering from Friedel oscillations and from proximity induced pair potential inside the QW. Here, all the four parameters are non-zero and flow under RG, as do the interaction parameters $\alpha$ and $\alpha^{\prime}$. An example where the system starts in the vicinity of the unstable fixed point SFP (as mentioned in Case IV in the Subsection. 3.1.2) is shown in Fig. 12. The NSN conductance here is defined as $G_{N S N}=G_{C A}-G_{C T}$. Here also we observe a strong non-monotonicity in the conductance which comes about due to the interplay of the electron and the hole channels taking part in transport, which contribute to the conductance with opposite signs, coupled with the effects from the RG flow of the interaction parameters.

\subsubsection{Non-ballistic FSN Junction}

In this case, for the ferromagnetic wire $r_{A}=0$, as for an up spin polarized QW, the incident electron can't pair up with a spin down electron in the same QW. On the other hand for the normal wire $r_{A}$ has a finite value. As explained earlier, the interaction parameters $\alpha$ and $\alpha^{\prime}$ on the ferromagnetic side do not renormalize, whereas they do on the normal side. Hence, even if we start from a situation where the interaction parameter $\alpha$ and $\alpha^{\prime}$ are symmetric for the two wires, RG flow will always give rise to an asymmetry in the interaction strength. Therefore, it becomes a very interesting case to study theoretically. The $\mathbb{S}$-matrix for this case has neither spin up-spin down symmetry, nor the wire index (left-right for two wires) symmetry. Only the particle-hole symmetry can be retained while parameterizing the $\mathbb{S}$-matrix. The latter case gets very complicated to study theoretically because the minimum number of independent complex-valued parameters that are required to parameterize the $\mathbb{S}$-matrix is nine as opposed to four in the NSN case. These are given by $r_{\uparrow \uparrow}^{11}, r_{\uparrow \uparrow}^{22}, r_{\downarrow \downarrow}^{22}, t_{A \uparrow \uparrow}^{12}, t_{A \downarrow \downarrow}^{21}, r_{A \uparrow \uparrow}^{22}, r_{A \downarrow \downarrow}^{22}, t_{\uparrow \uparrow}^{12}$, and $t_{\uparrow \uparrow}^{21}$. Here, 1(2) is the wire index for the ferromagnetic (normal) wire while, $\uparrow$ and $\downarrow$ are the respective spin polarization indices for the electron.

So, the minimal $\mathbb{S}$-matrix representing the FSN junction is given by

$$
\mathbb{S}=\left[\begin{array}{cccccc}
r & t & 0 & 0 & t_{A} & 0 \\
t^{\prime} & r^{\prime} & 0 & 0 & r_{A} & 0 \\
0 & 0 & r^{\prime \prime} & t_{A}^{\prime} & 0 & r_{A}^{\prime} \\
0 & 0 & t_{A} & r & 0 & t \\
t_{A}^{\prime} & r_{A}^{\prime} & 0 & 0 & r^{\prime \prime} & 0 \\
0 & 0 & r_{A} & t^{\prime} & 0 & r^{\prime}
\end{array}\right],
$$

In Eq. 59, we write down a representative $\mathbb{S}$-matrix which satisfies all the constraints of the FSN junction mentioned above as well as unitarity. We study its RG flow numerically by solving the nine coupled differential equations. The modulus of the S-matrix elements are given by $\left|r_{\uparrow \uparrow}^{11}\right|=\left|r_{\uparrow \uparrow}^{22}\right|=\left|r_{\downarrow \downarrow}^{22}\right|=\left|t_{A \uparrow \uparrow}^{12}\right|=\left|t_{A \downarrow \downarrow}^{21}\right|=\left|r_{A \uparrow \uparrow}^{22}\right|=$ $\left|r_{A \downarrow \downarrow}^{22}\right|=\left|t_{\uparrow \uparrow}^{12}\right|=\left|t_{\uparrow \uparrow}^{21}\right|=1 / \sqrt{3}$ and the corresponding phases associated with each of these amplitudes are $\pi / 3, \pi, 0,-\pi / 3,0, \pi / 3,0, \pi,-\pi / 3$ respectively. Here also we 


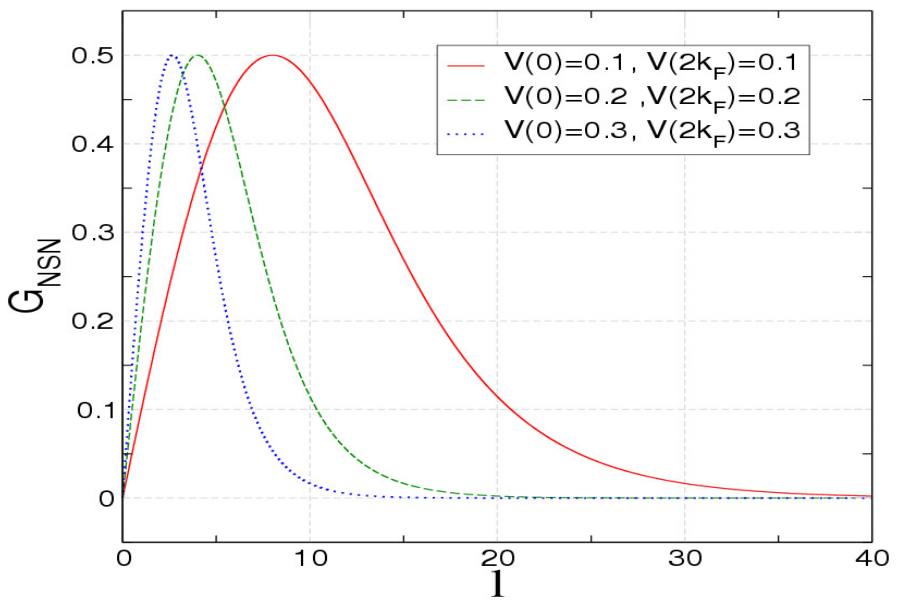

Fig. 12. (Color online) Conductance of the NSN junction $G_{N S N}=\left|t_{A}\right|^{2}-|t|^{2}$ is plotted in units of $2 e^{2} / h$ as a function of the dimensionless parameter $l$ where $l=\ln (L / d)$ and $L$ is either $L_{T}=\hbar v_{F} / k_{B} T$ at zero bias or $L_{V}=\hbar v_{F} / \mathrm{eV}$ at zero temperature and $d$ is the short distance cut-off for the RG flow. The three curves correspond to three different values of $V(0)$ and $V\left(2 k_{F}\right)$. Figure adapted from Ref. 75 .

observe a non-monotonic behavior of conductance, $G_{F S N}$ as a function of $l$ as shown in Fig. 13.

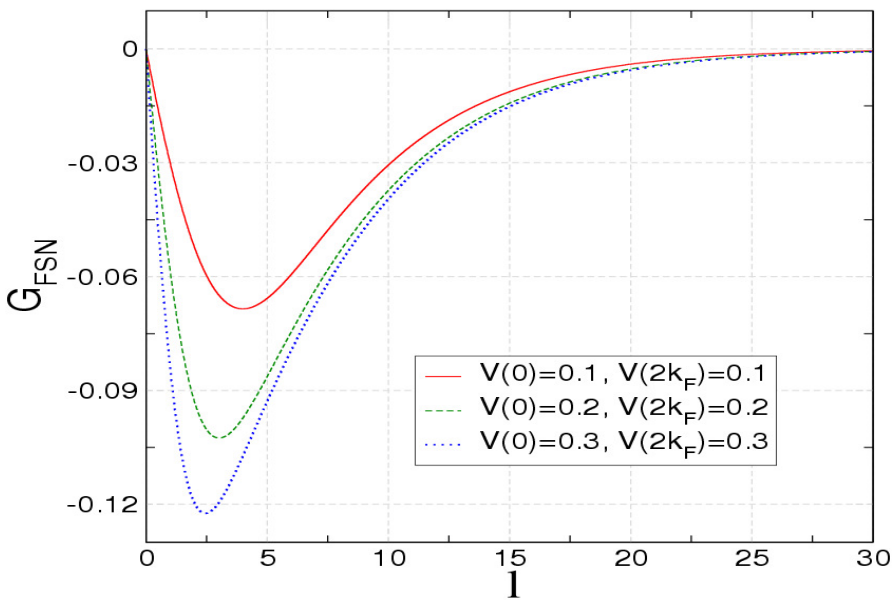

Fig. 13. (Color online) Charge conductance $G_{F S N}=\left|t_{A}\right|^{2}-|t|^{2}$ is plotted in units of $e^{2} / h$ for FSN junction as a function of the dimensionless parameter $l$ where $l=\ln (L / d)$ and $L$ is either $L_{T}=\hbar v_{F} / k_{B} T$ at zero bias or $L_{V}=\hbar v_{F} / e V$ at zero temperature. Here $d$ is the short distance cut-off for the RG flow. The three curves correspond to three different values of $V(0)$ and $V\left(2 k_{F}\right)$. Figure adapted from Ref. 75. 


\subsubsection{Non-ballistic FSF junction}

Here, we consider the case where both the wires are spin polarized. This case is similar to the ballistic case. Since here $r \neq 0$ and $r_{A}=0$, we will have the usual Friedel oscillations and the conductance will go to zero following the stable fixed point as a power law. Here again we have two instructive cases:

(a) When the two wires connected to the superconductor have their spin polarization aligned, i.e. $t \neq 0$, but $t_{A}=0$ and

(b) When the two wires have their spin polarization anti-aligned, i.e. $t=0$ but $t_{A} \neq 0$.

Both these cases are examples of case II of Subsubsection. 3.2.3. Either $t$ or $t_{A}$ need to be zero in the two cases mentioned above. Hence the parameters which are zero will remain zero under RG, while the non-zero parameters will flow according to Eqs. 47 and 48 respectively. The conductances are the same as in the NSN case except that the interaction parameters cannot flow now. The latter has already been emphasized in the insets in Figs. 10 and 11. Since the electrons are now effectively spin-less, $\alpha$ and $\alpha^{\prime}$ do not flow, and we get a monotonic fall-off of the conductance in both the cases.

The results of this section are summarized in the table below for the readers.

In this table we summarize all the relevant fixed points of the theory.

\begin{tabular}{|c|c|c|c|c|c|c|}
\hline$t$ & $t_{A}$ & $r_{A}$ & $r$ & Stability & Intermediate fixed point & Relevant physics \\
\hline 0 & 0 & 0 & 1 & Stable & $\times$ & RFP \\
\hline 0 & 0 & 1 & 0 & Unstable & $\times$ & AFP \\
\hline 0 & 1 & 0 & 0 & Unstable & $\times$ & CAFP \\
\hline 1 & 0 & 0 & 0 & Unstable & $\times$ & TFP \\
\hline $1 / 2$ & $1 / 2$ & $-1 / 2$ & $1 / 2$ & Unstable & $\sqrt{ }$ & SFP, Non-monotonic charge current \\
\hline$e^{i \phi_{1}} \sin \theta$ & $e^{i \phi_{2}} \cos \theta$ & 0 & 0 & Marginal & - & Pure spin current when $t=t_{A}$ \\
\hline
\end{tabular}

\subsubsection{Three wires-The Beam Splitter}

In this subsubsection, we consider the case of three QWs connected to a superconductor deposited on top of them. We assume that all the wires are connected within the phase coherence length of the superconductor. Hence, CAR can occur by pairing the incident electron with an electron from any of the other wires and emitting a hole in that wire. The conductance matrix can hence be extended for three wires as

$$
\left[\begin{array}{c}
I_{1} \\
I_{2} \\
I_{3}
\end{array}\right]=\left[\begin{array}{lll}
G_{r 11} & G_{t 12} & G_{t 13} \\
G_{t 12} & G_{r 22} & G_{t 23} \\
G_{t 13} & G_{t 23} & G_{r 33}
\end{array}\right]\left[\begin{array}{l}
V_{1} \\
V_{2} \\
V_{3}
\end{array}\right],
$$


with $\left(G_{C A i j}+G_{c t i j}\right) G_{r i j}=G_{A i i}+\sum_{j}\left(G_{C A i j}+G_{C T i j}\right)$ and $G_{t i j}=G_{C A i j}-G_{C T i j}$. Also the generalization to $N$ wires is obvious. Note that the conductances $G_{C A i j}=$ $G_{C A j i}$ and $G_{C T i j}=G_{C T j i}$. The relations of the conductances to the reflections and transmissions is obvious, for e.g., $G_{A i i} \propto\left|r_{A i i}\right|^{2}$ as before while $G_{C T i j} \propto\left|t_{i j}\right|^{2}$ and $G_{C A i j} \propto\left|t_{A i j}\right|^{2}$. The RG equations for the three wire case can be written using the matrix equation as given in Eq. 25 except that the $\mathbb{S}$-matrix is now $12 \times 12$ dimensional. For a system with particle-hole, spin up-spin down and wire index symmetry, the $\mathbb{S}$-matrix is given by,

$$
\mathbb{S}_{\uparrow}=\mathbb{S}_{\downarrow}=\left[\begin{array}{cccccc}
r & t & t^{\prime} & r_{A} & t_{A} & t_{A}^{\prime} \\
t & r & t & t_{A} & r_{A} & t_{A} \\
t^{\prime} & t & r & t_{A}^{\prime} & t_{A} & r_{A} \\
r_{A} & t_{A} & t_{A}^{\prime} & r & t & t^{\prime} \\
t_{A} & r_{A} & t_{A} & t & r & t \\
t_{A}^{\prime} & t_{A} & r_{A} & t^{\prime} & t & r
\end{array}\right],
$$

where we have chosen six independent parameters, with $t_{12}=t_{21}=t_{23}=t_{32}=t$ and $t_{13}=t_{31}=t^{\prime}$ and similarly for the CAR parameter $t_{A}$. The $F$ matrix now generalizes to

$$
F=\left[\begin{array}{cccccc}
\frac{\alpha r}{2} & 0 & 0 & \frac{-\alpha^{\prime} r_{A}}{2} & 0 & 0 \\
0 & \frac{\alpha r}{2} & 0 & 0 & \frac{-\alpha^{\prime} r_{A}}{2} & 0 \\
0 & 0 & \frac{\alpha r}{2} & 0 & 0 & \frac{-\alpha^{\prime} r_{A}}{2} \\
\frac{-\alpha^{\prime} r_{A}}{2} & 0 & 0 & \frac{\alpha r}{2} & 0 & 0 \\
0 & \frac{-\alpha^{\prime} r_{A}}{2} & 0 & 0 & \frac{\alpha r}{2} & 0 \\
0 & 0 & \frac{-\alpha^{\prime} r_{A}}{2} & 0 & 0 & \frac{\alpha r}{2}
\end{array}\right]
$$

There exists possibility of emergence of many more non-trivial fixed points in this case. For instance, the AGFP, as mentioned in Subsubsection. 3.1.4. As discussed in Subsubsection. 3.1.4, for the reflection-less case with symmetry between just two wires, this complicated $\mathbb{S}$-matrix described by Eq. 61 takes a very simple form, which can be dealt analytically. Within the sub-space considered we found that the AGFP was a stable fixed point. In Fig. 14, we show the RG flow of $\left|t_{A}\right|^{2}$ from two different unstable fixed points to the stable AGFP.

The possibility of experimental detection of such a non-trivial fixed point with intermediate transmission and reflection is quite interesting. From this point of view, the AGFP is a very well-suited candidate as opposed to its counterpart, the Griffith's fixed point ${ }^{27,65}$. For a normal junction of three $1-\mathrm{D} Q \mathrm{QW}$, the $\mathbb{S}$-matrix corresponding to $r=-1 / 3, t=2 / 3$ is a fixed point (GFP), where $r$ and $t$ are the reflection and the transmission for a completely symmetric three wire junction. Even though it is an interesting fixed point, it turns out to be a repulsive one and hence the possibility of its experimental detection is very low. On the contrary, the AGFP, being an attractive fixed point, has a better possibility of being experimentally measured. The main point here is that even if we begin with an asymmetric junction, which 
is natural in a realistic experimental situation, the effect of interaction correlations inside the QWs are such that as we go down in temperature, the system will flow towards the symmetric junction. This feature can be inferred from the results shown in Fig. 14.

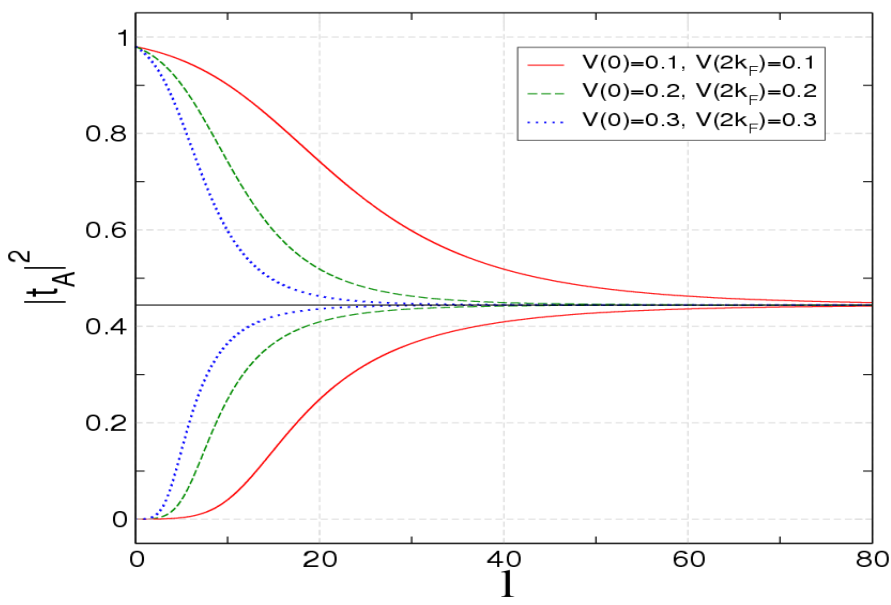

Fig. 14. $\left|t_{A}\right|^{2}$ is plotted as a function of dimensionless parameter $l$ where $l=\ln (L / d)$ and $L$ is either $L_{T}=\hbar v_{F} / k_{B} T$ at zero bias or $L_{V}=\hbar v_{F} / e V$ at zero temperature and $d$ is the short distance cut-off for the RG flow. The three curves correspond to three different values of $V(0)$ and $V\left(2 k_{F}\right)$. The set of curves in the top represent the RG flow of $\left|t_{A}\right|^{2}$ when the starting point is in the vicinity of $r_{A}=0$ fixed point while, the set of curves in the bottom half represent the RG flow of $\left|t_{A}\right|^{2}$ with starting point close to $r_{A}=-1$. Figure adapted from Ref. 75 .

\section{Stability analysis of the RG flow for the NSN junction of LL wires}

In this section we study the RG flows of the two terminal conductance of a superconducting junction of two LL wires ${ }^{77}$. In particular we perform the stability analysis of the various fixed points of the theory discussed in Sec. 3 and compute the power laws associated with the RG flow around them. Our analysis also provides the renormalized values of the various transmission and reflection amplitudes around these fixed point values which can then be used to obtain the LB conductances.

\subsection{Method of stability analysis}

To carry out our stability analysis we adopt the RG method followed in Sec. 2 and 3 where an $\mathbb{S}$-matrix formulation was used to compute the linear conductance and inter-electron interactions inside the QW were taken into account by allowing the S-matrix to flow as a function of the relevant energy scale (like temperature, bias voltage or system size) using an RG procedure. This method works well when e-e interaction strength inside the QW is weak so that it can be treated perturbatively. 
In our stability analysis we mainly focus on three different fixed points- the chiral fixed point (CFP) and GFP of a normal junction of three LL wires ${ }^{27,99}$ and the SFP ${ }^{75}$ of the NSN junction also introduced in Sec. 3. First we discuss the stability around the CFP and GFP of a normal junction of three LL wires (Y-junction) to benchmark our calculation with known results ${ }^{27}$. As a first step towards performing a systematic stability analysis, we need to obtain an $\mathbb{S}$-matrix which results from a very small unitary deviation around the fixed point $\mathbb{S}$-matrix. Given the number of independent parameters of the $\mathbb{S}$-matrix dictated by symmetry and unitarity constraints, the most general deviation from the fixed point $\mathbb{S}$-matrix can be obtained by multiplying the fixed point $\mathbb{S}$-matrix by another unitary matrix which is such that it allows for a straightforward expansion in terms of small parameters around the identity matrix. The latter is realized as follows-

$$
\mathbb{S}=\mathbb{S}_{0} \exp \left\{i \sum_{j=1}^{9} \epsilon_{j} \lambda_{j}\right\},
$$

where $\mathbb{S}_{0}$ represents the fixed point $\mathbb{S}$-matrix and $\lambda_{j}$ 's (along with the identity $\left.\lambda_{0}=I\right)$ are the eight generators of the $S U(3)$ group which are traceless hermitian matrices. This can be straightforwardly generalized to the case of $N$ wires by using $S U(N)$ matrices. Perturbations around these fixed points are characterized in terms of the $\epsilon_{j}$ 's. Of course, the resulting $\mathbb{S}$-matrix obtained in this way corresponds to a small unitary deviation when $\epsilon_{j}$ 's are small parameters. To first order in $\epsilon_{j}$ 's, Eq. 63 reduces to

$$
\mathbb{S}=\mathbb{S}_{0}\left(\mathbb{I}+i \sum_{j} \epsilon_{j} \lambda_{j}\right),
$$

where $\mathbb{S}_{0}$ for CFP and GFP fixed points are given by ${ }^{27}$

$$
\mathbb{S}_{0}^{C F P}=\left[\begin{array}{lll}
0 & 1 & 0 \\
0 & 0 & 1 \\
1 & 0 & 0
\end{array}\right] ; \mathbb{S}_{0}^{G F P}=\left[\begin{array}{ccc}
-1 / 3 & 2 / 3 & 2 / 3 \\
2 / 3 & -1 / 3 & 2 / 3 \\
2 / 3 & 2 / 3 & -1 / 3
\end{array}\right],
$$

respectively. Using Eq. 64, the RG equation (Eq. 20 given in Sec. 2) with $\mathbb{S}$ expanded to the linear order in $\epsilon_{j}$ becomes

$$
i \sum_{j=1}^{9} \lambda_{j} \frac{d \epsilon_{j}}{d l}=\mathbb{S}_{0}^{\dagger}\left[\mathbb{I}-i \sum_{j} \epsilon_{j} \lambda_{j}\right]\left\{\mathbb{F}-\mathbb{S}_{0}\left[\mathbb{I}+i \sum_{j} \epsilon_{j} \lambda_{j}\right] \mathbb{F}^{\dagger}\left[\mathbb{I}+i \sum_{j} \epsilon_{j} \lambda_{j}\right]\right\}
$$

where $\mathbb{F}$ is the diagonal part of the following quantity

$$
\mathbb{F}=\frac{\alpha}{2} \mathbb{S}_{0}\left[\mathbb{I}+i \sum_{j} \epsilon_{j} \lambda_{j}\right]_{\text {diagonal }} .
$$

By restricting the RHS of Eq. 66 to linear order in $\epsilon_{j}$ 's, one then obtains nine coupled linear differential equations. At the next step, by applying a unitary rotation, we 
can decouple these coupled equations (Eq. 66) and re-cast them in terms of new variables $\epsilon_{j}^{\prime}$ (which are linear combinations of the original $\epsilon_{j}$ ). The equations are now given by

$$
\frac{d \epsilon_{j}^{\prime}}{d l}=\mu_{j} \epsilon_{j}^{\prime}
$$

where $\mu_{j}$ is a real number corresponding to the 'power law' associated with perturbations turned on along each of the new nine eigen-directions $\epsilon_{j}^{\prime} . \mu_{j}<0$ indicates that the given direction is stable and $\mu_{j}>0$ indicates that it is unstable. Here the non-diagonal $\epsilon_{j}$ are related to the diagonal $\epsilon_{j}^{\prime}$ by $\epsilon_{j}=\sum_{i} \mathbb{U}_{j i} \epsilon_{i}^{\prime}$ where $\mathbb{U}$ is the diagonalizing rotation matrix.

\subsection{Power laws around different fixed points}

Using the method discussed above we obtain all the power laws associated with the independent perturbations that can be switched on around a given fixed point $\mathbb{S}$-matrix. Now it is straightforward to show that the power laws associated with the CFP and GFP are given by $[\alpha / 2, \alpha / 2,0, \alpha / 2, \alpha / 2, \alpha / 2, \alpha / 2,0,0]$ and $[0,0,0$, $0,0,-\alpha / 3,2 \alpha / 3,2 \alpha / 3, \alpha]$ respectively which is consistent with results obtained in Ref. $27^{\mathrm{a}}$. The value zero corresponds to marginal directions while the values with positive or negative signs correspond to stable or unstable directions respectively. We do not write the explicit form of the $\mathbb{U}$ matrix for the CFP and GFP as they are needed only for obtaining the explicit form of the power law correction to the fixed point conductance which is beyond the scope of this review.

Finally, let us discuss the stability around the different RG fixed points of the NSN junction. First we focus on the SFP of the NSN junction which has intermediate reflections and transmissions. As discussed in Sec 3 in presence of e-e interaction inside the QW the incident electron (hole) not only scatters from the Friedel oscillations as an electron (hole) but also scatters from the proximity induced pair potential inside the QW as a hole (electron). Now the amplitude of both of these scattering processes are proportional to the e-e interaction strength inside the QW. The interplay between these two scattering processes which actually arise due to the e-e interaction strength inside the QW, renormalize the bare scattering amplitudes at the junction and give rise to this new SFP where all the scattering amplitudes have intermediate non-zero values. This fact is unique about this fixed point and hence this fixed point is the central focus of our discussion here.

We adopt the same procedure as described above for the three-wire junction but now with $S U(4)$ generators. This is so because the full $8 \times 8 \mathbb{S}$-matrix describing the NSN junction has a block diagonal form with each spin block (up and down

\footnotetext{
a There is a correction to the power laws obtained for the GFP for the three-wire junction in Ref. 27. Lal et al. had predicted a stable direction with power law $-\alpha$ which should be corrected to $-\alpha / 3^{100}$ as is obtained in this section. The corrected power law is also consistent with that obtained in Ref. 99 using a functional RG procedure.
} 
spin sectors) being represented by a $4 \times 4$ matrix. Hence we have a unitary starting $\mathbb{S}$-matrix deviating from the fixed point $\mathbb{S}$-matrix $\left(\mathbb{S}_{0}\right)$, as given before by Eq. 64 , except that now the sum over $j$ runs from 1 to 16 since $\lambda_{j}$ 's now represent the fifteen generators of the $S U(4)$ group along with the identity matrix. The $\mathbb{S}_{0}$ which describes the SFP ${ }^{75}$ is given by $r=1 / 2, t=1 / 2, r_{A}=-1 / 2$ and $t_{A}=1 / 2$. Note that the SFP is a particle-hole, left-right symmetric fixed point and hence the entire $4 \times 4 \mathbb{S}$-matrix is determined completely by the above given four amplitudes for $r, t, r_{A}, t_{A}$.

We then solve Eq. 66 for the present case with sixteen coupled equations up to the first order in the small perturbations characterized by $\epsilon_{j}$ 's. We obtain sixteen eigenvalues which correspond to the power laws around the different sixteen eigendirections. These power laws around the various eigen-directions can be listed as $[0$, $0,0,0,0,-\alpha / 2,-\alpha / 2,\left(\alpha-\alpha^{\prime}\right) / 2, \alpha^{\prime} / 2, \alpha^{\prime} / 2,\left(-\alpha+\alpha^{\prime}\right) / 2,\left(-\alpha+\alpha^{\prime}\right) / 2,\left(\alpha+\alpha^{\prime}\right) / 2$, $\left.\left(\alpha+\alpha^{\prime}\right) / 2,\left(\alpha-\alpha^{\prime}-\sqrt{9 \alpha^{2}+14 \alpha \alpha^{\prime}+9 \alpha^{\prime 2}}\right) / 4,\left(\alpha-\alpha^{\prime}+\sqrt{9 \alpha^{2}+14 \alpha \alpha^{\prime}+9 \alpha^{\prime 2}}\right) / 4\right]$. Hence note that there are five marginal directions, two stable directions, four unstable directions and four other directions whose stability depends on the sign of $\alpha-\alpha^{\prime}$. One of the most striking outcomes of this analysis is the fact that we obtain two power laws which are not just simple linear combinations of $V(0)$ and $V\left(2 k_{F}\right)$. Instead, they appear as square roots of quadratic sum of these quantities. Our analysis actually leads to the first demonstration of the existence of such power laws in the context of quantum impurity problems in LL theory and this is the central result of this section.

Having obtained the power laws around SFP, the next task is to obtain an explicit expression for the Landauer-Buttiker conductance corresponding to perturbations around these fixed points along some of the eigen-directions. Now note that the RG equation is expressed in terms of $\epsilon^{\prime}$ 's whereas the $\mathbb{S}$-matrix representing small deviations from the fixed point is expressed in terms of $\epsilon$ 's. The two terminal linear conductance across the junction depends explicitly on the $\mathbb{S}$-matrix element which are expressed in terms of $\epsilon$ 's (see Eq. 64). Hence in order to obtain an expression for conductance in terms of the temperature or the applied bias voltage dependence induced by e-e interaction, we need to first assign bare values to the various perturbations parameterized by $\epsilon^{\prime}$ s and then express the $\epsilon^{\prime \prime}$ s evolved under RG flow in terms of these bare values of $\epsilon^{\prime}$ 's as $\epsilon^{\prime}(\Lambda)=\left(\Lambda / \Lambda_{0}\right)^{\mu} \epsilon_{0}^{\prime}$ where $\Lambda$ corresponds to the energy scale at which we are probing the system (which can be either voltage bias at zero temperature or temperature at vanishing bias voltage) and $\Lambda_{0}$ is the high energy cut-off expressed in terms of voltage or temperature. Then by using the rotation matrix which diagonalizes the coupled RG equations, we express $\epsilon$ 's in terms of $\epsilon^{\prime \prime}$ s written explicitly as a function of temperature or voltage. Finally plugging these renormalized values of $\epsilon$ 's into the $\mathbb{S}$-matrix given by Eq. 64, we get all the transmission and reflection amplitudes for the system as explicit functions of the temperature or voltage carrying the specific power laws associated with perturbations switched on along the eigen-directions. These amplitudes are now directly 
related to the linear conductances.

Now we calculate expression for conductance for a simple case where only one of the $\epsilon^{\prime}\left(=\epsilon_{15}^{\prime}\right)$ is turned on. For this particular case we need the $\mathbb{U}$ matrix which is given by

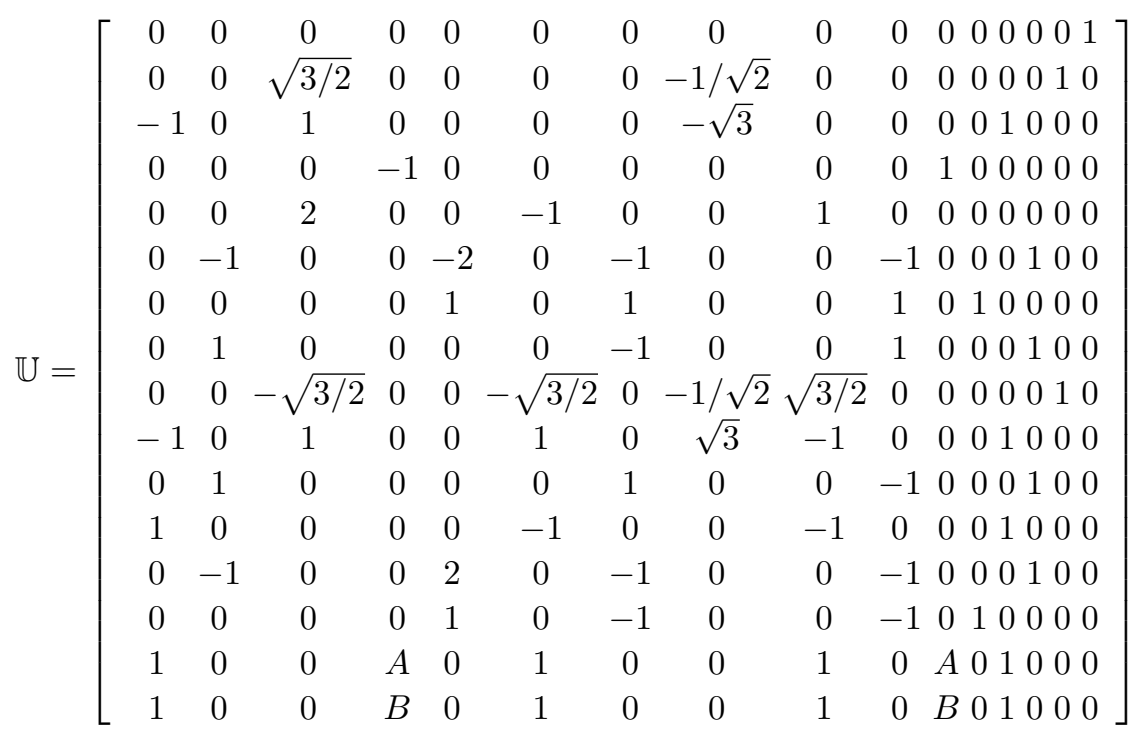

where, $A=4\left(\alpha+\alpha^{\prime}\right) /\left(\alpha-\alpha^{\prime}-\sqrt{9 \alpha^{2}+14 \alpha \alpha^{\prime}+9 \alpha^{\prime 2}}\right) / 4 \quad$ and $B=4\left(\alpha+\alpha^{\prime}\right) /\left(\alpha-\alpha^{\prime}+\sqrt{9 \alpha^{2}+14 \alpha \alpha^{\prime}+9 \alpha^{\prime 2}}\right) / 4$.

We choose this specific direction to perturb the system as this corresponds to a power law which is not a linear function of $V(0)$ and $V\left(2 K_{F}\right)$ and hence interesting to study. The $\mathbb{S}$-matrix to quadratic order in $\epsilon_{15}^{\prime}$ is given by

$$
\mathbb{S}=\left[\begin{array}{llll}
\frac{\left[1-(1-i) \epsilon_{15}^{\prime}-\epsilon_{15}^{\prime}{ }^{2}\right]}{2} & \frac{\left[1+\epsilon_{15}^{\prime}\right]}{2}-\frac{[1-i] \epsilon_{15}^{\prime}{ }^{2}}{4} & -\frac{1}{2} & \frac{\left[1+i \epsilon_{15}^{\prime}\right]}{2}-\frac{[1+i] \epsilon_{15}^{2}}{4} \\
\frac{\left[1-(1+i) \epsilon_{15}^{\prime}-\epsilon_{15}^{\prime}{ }^{2}\right]}{2} & \frac{\left[1+\epsilon_{15}^{\prime}\right]}{2}-\frac{[1+i] \epsilon_{15}^{\prime}{ }^{2}}{4} & \frac{1}{2} & \frac{\left[1-i \epsilon_{15}^{\prime}\right]}{2}+\frac{[1-i] \epsilon_{15}^{\prime}}{4} \\
-\frac{\left[1+(1-i) \epsilon_{15}^{\prime}-\epsilon_{15}^{\prime}{ }^{2}\right]}{2} & \frac{\left[1-\epsilon_{15}^{\prime}\right]}{2}-\frac{[1-i] \epsilon_{15}^{\prime}}{4} & \frac{1}{2} & \frac{\left[1-i \epsilon_{15}^{\prime}\right]}{2}-\frac{[1-i] \epsilon_{15}^{\prime}}{4} \\
\frac{\left[1+(1+i) \epsilon_{15}^{\prime}-\epsilon_{15}^{\prime}{ }^{2}\right]}{2} & -\frac{\left[1-\epsilon_{15}^{\prime}\right]}{2}+\frac{[1+i] \epsilon_{15}^{2}}{4} & \frac{1}{2} & \frac{\left[1+i \epsilon_{15}^{\prime}\right]}{2}+\frac{[1-i] \epsilon_{15}^{2}}{4}
\end{array}\right],
$$

So, the scaling of sub-gap conductance (to $\mathcal{O}\left(\epsilon_{15}^{\prime 2}\right)$ ) for an incident electron and a hole taking into account both spin-up and spin-down contributions in units of $2 e^{2} / h$ is given by

$$
G_{12}^{e}=-\frac{\epsilon_{15}^{\prime}}{2} ; G_{21}^{e}=\frac{\epsilon_{15}^{\prime}}{2},
$$




$$
G_{12}^{h}=-\frac{\epsilon_{15}^{\prime}}{2} ; G_{21}^{h}=\frac{\epsilon_{15}^{\prime}}{2}
$$

where $\epsilon_{15}^{\prime}=\epsilon_{15,0}^{\prime}\left(\Lambda / \Lambda_{0}\right)^{\left(\alpha-\alpha^{\prime}-\sqrt{9 \alpha^{2}+14 \alpha \alpha^{\prime}+9 \alpha^{\prime 2}}\right) / 4}$. Here the superscripts $e$ and $h$ stand for electrons and holes while the subscripts 1 and 2 stand for first and second QW respectively. Also, $G_{12}^{e}=\left|t_{A, 12}^{e h}\right|^{2}-\left|t_{12}^{e e}\right|^{2}$ where $t^{e e}$ is the transmission amplitude for electrons and $t_{A}^{e h}$ represents CAR amplitude for electrons. Similar expressions also hold for the holes. In the expressions of power laws given above, $\alpha=\left(g_{2}-\right.$ $\left.2 g_{1}\right) / 2 \pi \hbar v_{F}$ and $\alpha^{\prime}=\left(g_{1}+g_{2}\right) / 2 \pi \hbar v_{F}$ where the bare values of $g_{1}(d)=V\left(2 k_{F}\right)$ and $g_{2}(d)=V(0)$. In our stability analysis, we have assumed $\alpha<\alpha^{\prime}$ which is consistent with experimental observations ${ }^{4}$. For the special case when $g_{2}=2 g_{1}, \alpha$ vanishes and only $\alpha^{\prime}$ survives.

It is very interesting to note that even though the $\mathbb{S}$-matrix corresponding to perturbation along $\epsilon_{15}^{\prime}$ breaks both time reversal and electron-hole symmetry, the two terminal linear conductance restores particle-hole symmetry. Secondly it might be of interest to note the fact that the fixed point conductance admits correction along $\epsilon_{15}^{\prime}$ which is linear in $\epsilon_{15}^{\prime}$ and not quadratic. Normally when we perform a stability analysis around a fixed point $\mathbb{S}$-matrix whose elements are constituted out of unimodular numbers (representing disconnected or perfectly connected fixed points), it is always possible to identify various terms of the $\mathbb{S}$-matrix, representing small unitary deviations from the fixed point $\mathbb{S}_{0}$-matrix in terms of various tunneling operators which are perturbatively turned on around the fixed point Hamiltonian. Hence a straight forward perturbative linear conductance calculation using the Hamiltonian along with the tunneling parts will suggest that the correction due to the $\mathbb{S}$-matrix representing small deviation from fixed point $\mathbb{S}_{0}$-matrix must introduce correction to fixed point conductance which are quadratic in terms of the deviation parameter. Although this argument applies only to those fixed points which correspond to completely connected or disconnected wires and not applicable to fixed points which have intermediate values for various transmission and reflection amplitudes like the SFP. In other words, an arbitrary deviation from SFP may not be easily representable as a tunneling operator. This argument explains why the linear dependence of the conductance on $\epsilon^{\prime}$ and hence the corresponding power law dependence looks unconventional.

As a cross check, we observe that we get back the power laws associated with the symmetric fixed point ${ }^{27}$ of the four-wire junction once we substitute $\alpha^{\prime}=0$ in the expression for the power laws of the SFP for the NSN junction. Although our geometry does not correspond to the real junction of four QWs, the presence of both electron and hole channel mimics the situation of a four-wire junction. More specifically, the SFP of the NSN junction turns out to be identical to the symmetric fixed point of the four-wire junction due to perfect particle-hole symmetry of the SFP when $\alpha^{\prime}$ is set equal to zero.

Here we enumerate and discuss the stability of the other fixed points (RFP, AFP, TFP and CAFP) discussed earlier in Sec 3 as well as obtained in Ref. 75 for 
the NSN junction :

(a) $t=t_{A}=r_{A}=0, r=1$ (RFP) : This fixed point turns out to be stable against perturbations in all directions. There are ten directions for which the exponent is $-\alpha$ while two others with the exponents $-\left(\alpha+\alpha^{\prime}\right)$. The remaining four directions are marginal.

(b) $t=t_{A}=r=0, r_{A}=1$ (AFP) : This is unstable against perturbations in twelve directions. There are ten directions with exponent $\alpha$ and two directions with exponent $\left(\alpha+\alpha^{\prime}\right)$. The remaining four directions are marginal, as for RFP.

(c) $r_{A}=t_{A}=r=0, t=1$ (TFP) : This fixed point has four unstable directions with exponent $\alpha$, two stable directions with the exponent $-\alpha^{\prime}$ and the remaining directions are marginal.

(d) $r_{A}=t=r=0, t_{A}=1$ (CAFP) : This has four unstable directions with exponent $\alpha$ and two stable directions with the exponent $-\alpha^{\prime}$ and the remaining directions are marginal.

Note that the close similarity in stability between CAFP and TFP fixed points can be attributed to the fact that both these fixed points belong to the continuous family of marginal fixed points defined by the condition $|t|^{2}+\left|t_{A}\right|^{2}=1$. The entire family of fixed points is marginal because for these fixed points, the amplitudes for Friedel oscillation and pair potential in the QWs vanish identically.

Hence, we notice that for the AFP only the scattering amplitude from the pair potential inside the $\mathrm{QW}$ is non-zero as only $r_{A}$ is non-zero. On the other hand for RFP only the scattering amplitude from Friedel oscillations are non-zero as only $r$ is nonzero. Furthermore, both for CAFP and TFP, the amplitude for scattering from the Friedel oscillations as well as from the pair potential is zero as in these cases both $r$ and $r_{A}$ are zero. Hence SFP is the only fixed point for which both the amplitude for scattering from the Friedel oscillations and the pair potential are finite; hence, this fixed point is nontrivial and elegant in the literature of LL wires. Its very existence can be attributed to the interplay of these two different scattering processes arising from Friedel oscillations and the pair potential inside the QW. The two terminal conductance at this fixed point gets contribution from both the CT of electrons through the superconductor as well as through the non local CAR process. Since both electron and hole channels contribute with opposite signs to conductance, if we apply a small perturbation around this fixed point, we get an interesting nonmonotonic behavior of the conductance $G_{\mathrm{NSN}}=G_{\mathrm{CAR}}-G_{\mathrm{CT}}$ as depicted in Fig. 12. This effect emerges due to the competition between the electron and the hole channels and it can be of interest from an experimental point of view as well as application point of view which we discuss in the next section. 


\section{Spintronics with NSN Junction of 1-D quantum wires}

Two fundamental degrees of freedom associated with an electron that are of direct interest to condensed matter physics are its charge and spin. Until recent times, all conventional electron-based devices have been solely based upon the utilization and manipulation of the charge degree of freedom of an electron. However, the realization of the fact that devices based on the spin degree of freedom can be almost dissipation-less and with very fast switching times, has led to an upsurge in research activity in this direction from the last decade ${ }^{101,102,103}$. The first step towards realization of spin-based electronics (spintronics) would be to produce pure spin current (SC).

In this section we demonstrate possible scenarios for production of pure SC and large tunnelling magnetoresistance (TMR) ratios from CT and CAR across a superconducting junction comprising of normal metal-superconductor-normal metal (NSN), where, the normal metal is a 1-D interacting QW. Here we demonstrate that some of the fixed points associated with the NSN junction of 1-D QW discussed in Sec. 3 correspond to the case of pure SC. We also analyze the influence of e-e interaction and see how it stabilizes or de-stabilizes the production of pure SC.

Formally, it is straightforward to define a charge current as a product of local charge density with the charge velocity, but such a definition cannot be straightforwardly extended to the case of SC. This is because both spin $\vec{S}$ and velocity $\vec{v}$ are vector quantities and hence the product of two such vectors will be a tensor. In our study, we adopt the simple minded definition of SC, which is commonly used ${ }^{102,104}$. It is just the product of the local spin polarization density associated with the electron or hole, (a scalar $s$ which is either positive for up-spin or negative for down-spin) and its velocity ${ }^{102,104}$. The most obvious scenario in which one can generate a pure SC in the way defined above would be to have (a) an equal and opposite flow of identically spin-polarized electrons through a channel, such that the net charge current through the channel is nullified leaving behind a pure SC, or (b) alternatively, an equal flow of identically spin polarized electrons and holes in the same direction through a channel giving rise to pure SC with perfect cancellation of charge current. In this section, we explore the second possibility for generating pure SC using a NSN junction of 1-D QWs.

\subsection{Proposed device and its theoretical modelling}

The configuration we have in mind for the production of pure SC is shown in Fig. 15. The idea is to induce a pair potential in a small region of a quantum wire (QW) by depositing a superconducting strip on top of the wire (which may be, for instance, a carbon nanotube or $G a A s \mathrm{QW}$ ) due to proximity effects. If the strip width on the wire is of the order of the phase coherence length of the superconductor, then both direct CT as well as CAR to hole tunnelling can occur across the superconducting region ${ }^{40,41}$. It is worth pointing out that in the case of a singlet superconductor, which is the case we consider, both the tunnelling processes will conserve spin. In 


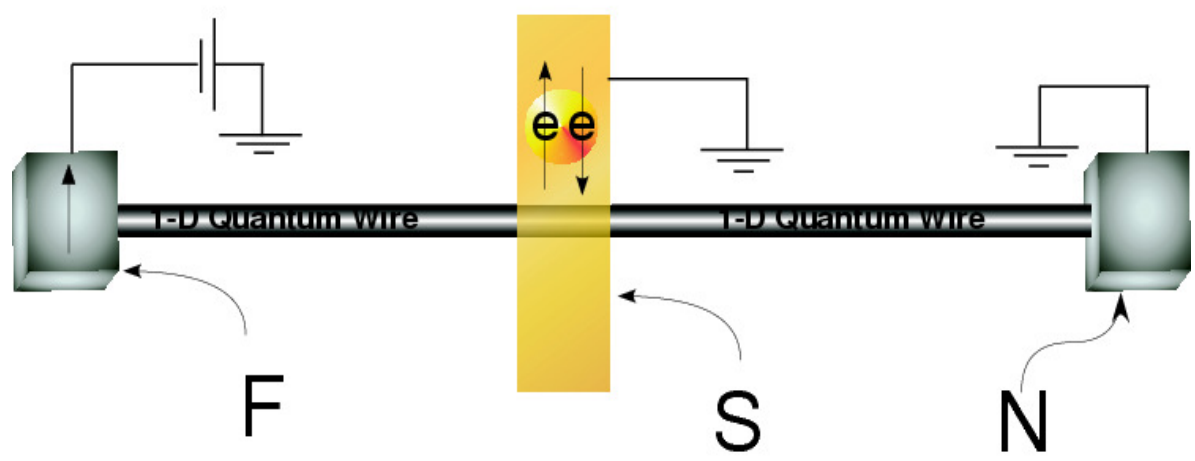

Fig. 15. (Color online) A 1-D quantum wire (carbon nanotube) connected to a ferromagnetic (F) lead on the left and a normal (N) lead on the right. The thin strip in the middle of the QW depicts a 2-D layer of bulk superconducting material deposited on top of the wire. Figure adapted from Ref. 76.

order to describe the mode of operation of the device (see Fig. 15), we first assume that the $\mathbb{S}$-matrix representing the NSN junction described above respects parity symmetry about the junction, particle-hole symmetry and spin-rotation symmetry. Considering all the symmetries, we can describe the superconducting junction connecting the two half wires by an $\mathbb{S}$-matrix with only four independent parameters namely, (i) the normal refection amplitude $(r)$ for e (h), (ii) the transmission amplitude $(t)$ for e $(\mathrm{h})$, (iii) AR amplitude $\left(r_{A}\right)$ for e $(\mathrm{h})$, and (iv) the CAR amplitude $\left(t_{A}\right)$ for e (h). If we inject spin polarized electron $(\uparrow \mathrm{e})$ from the left QW using a ferromagnetic contact ${ }^{113}$ and tune the junction parameters such that $t$ and $t_{A}$ are equal to each other, it will lead to a pure SC flowing in the right QW (see Fig. 15). This is so because, on an average, an equal number of electrons ( $\uparrow$ e) (direct electron to electron tunnelling) and holes $(\uparrow \mathrm{h}$ ) (crossed Andreev electron to hole tunnelling) are injected from the left wire to the right wire resulting in production of pure SC in the right wire. Note that spin up holes $(\uparrow \mathrm{h})$ implies a Fermi sea with an absence of spin down electron (which is what is needed for the incident electron ( $\uparrow \mathrm{e}$ ) to form a Cooper pair and enter into the singlet superconductor). Generation of pure SC by superconducting proximity effect has also been proposed very recently in the context of $\mathcal{T}$-stub geometry ${ }^{108}$, superconducting double barrier structure ${ }^{109}$, interacting quantum dots ${ }^{110}$ and ferromagnetic quantum point contact ${ }^{111}$.

\subsection{Results for Spin current and Tunneling magnetoresistance}

To discuss pure SC in NSN junction we propose two possible $\mathbb{S}$-matrices $\left(\mathbb{S}_{1}\right.$ and $\mathbb{S}_{2}$ ) that can be realized within our set-up which will finally lead to the production of pure SC. The spin conductance is defined as $G_{\uparrow}^{S}\left(G_{\downarrow}^{S}\right) \propto|t|^{2}+\left|t_{A}\right|^{2}$ whereas the charge conductance is given by $G_{\uparrow}^{C}\left(G_{\downarrow}^{C}\right) \propto-\left(|t|^{2}-\left|t_{A}\right|^{2}\right)$. The $\uparrow$ and $\downarrow$ arrows in the subscript represent the spin polarization of the injected electrons from the ferromag- 


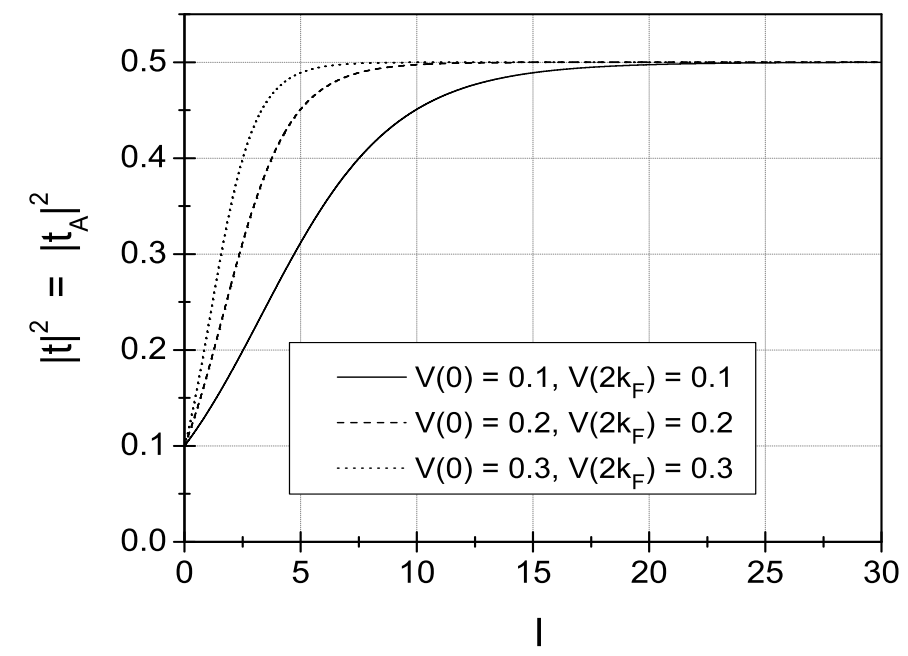

Fig. 16. (Color online) The variation of $|t|^{2}\left(=\left|t_{A}\right|^{2}\right)$ is plotted as a function of the dimensionless parameter $l$ where $l=\ln (L / d)$ and $L$ is either $L_{T}=\hbar v_{F} / k_{B} T$ at zero bias or $L_{V}=\hbar v_{F} / e V$ at zero temperature and $d$ is the short distance cut-off for the RG flow. The three curves correspond to the three different values of $V(0)$ and $V\left(2 k_{F}\right)$ for the NSN junction. This case corresponds to the $\mathbb{S}$-matrix given by $\mathbb{S}_{1}$. Figure adapted from Ref. 76 .

netic reservoir (see Fig. 15). The negative sign in the expression for $G_{\uparrow}^{C}\left(G_{\downarrow}^{C}\right)$ arises because it is a sum of contribution coming from two oppositely charged particles (electrons and holes). The first $\mathbb{S}$-matrix, $\mathbb{S}_{1}$ has $r=0$ (reflection-less), $r_{A} \neq 0$ and $t=t_{A}$. The latter is not a fixed point as listed in Sec. 3 and hence the parameters of the $\mathbb{S}$-matrix flows under the RG scheme described in Subsec. 3.1. It is easy to see from Eqs. 45 - 48, that for this case, the RG equations for $t$ and $t_{A}$ are identical, and hence it is ensured that the RG flow will retain the equality of the $t$ and $t_{A}$ leading to the preservation of pure SC. Physically this implies that of a situation in which if we start the experiment with this given $\mathbb{S}$-matrix $\left(\mathbb{S}_{1}\right)$ at the high energy scale (at finite bias voltage and zero temperature or at zero bias and finite temperature), then, as we reduce the bias in the zero temperature case (or reduce the temperature in the zero bias case), the correlations arising due to inter-electron interactions in the wire are such that the amplitude of $t$ and $t_{A}$ will remain equal to each other. The quantity which increases with increasing length scale $L$ is the absolute value of the amplitude $t$ or $t_{A}$ leading to a monotonic increase of pure SC till it saturates at the maximum value allowed by the symmetries of the $\mathbb{S}$-matrix, $\mathbb{S}_{1}$ (depicted in Fig. 16). Here all the $\mathbb{S}$-matrix elements are assumed to be energy independent and hence the bias dependence is solely due to RG flow. Of course the bias window has to be small enough so that the energy dependence of $t, t_{A}, r$ and $r_{A}$ can be safely ignored. This saturation point is actually a stable fixed point of the theory if the 
junction remains reflection-less $(r=0)$. So we observe that the transmission (both $t$ and $t_{A}$ ) increases to maximum value while the AR amplitude scales down to zero leading to pure SC. Note that the interaction induced correction enhances the amplitude for pure SC and also stabilizes the pure SC operating point. This makes the operating point, $\mathbb{S}_{1}$ quite well-suited for an experimental situation. Fig. 16 shows the variation of the pure spin conductance $\left(=2 \times|t|^{2}\right.$ in units of $\left.e^{2} / h\right)$ as a function
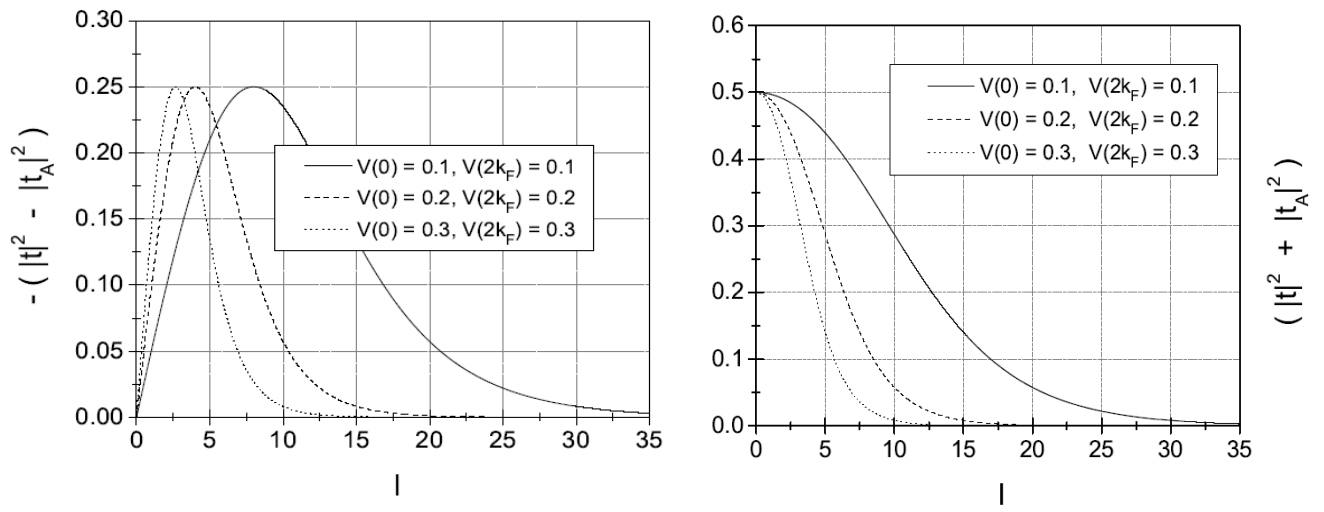

Fig. 17. (Color online) The variation of $-\left(|t|^{2}-\left|t_{A}\right|^{2}\right) \propto G_{\uparrow}^{C}$ or $G_{\downarrow}^{C}$ and the variation of $\left(|t|^{2}+\right.$ $\left.\left|t_{A}\right|^{2}\right) \propto G_{\uparrow}^{S}$ or $G_{\downarrow}^{S}$ are plotted as a function of the dimensionless parameter $l$ where $l=\ln (L / d)$ and $L$ is either $L_{T}^{\downarrow}=\hbar v_{F} / k_{B} T$ at zero bias or $L_{V}=\hbar v_{F} / e V$ at zero temperature and $d$ is the short distance cut-off for the RG flow. The three curves in each plot correspond to three different values of the interaction parameter $V(0)$ and $V\left(2 k_{F}\right)$ for the NSN junction. These plots correspond to the $\mathbb{S}$-matrix given by $\mathbb{S}_{2}$. Figure adapted from Ref. 76 .

The second case corresponds to the most symmetric $\mathbb{S}$-matrix $\left(\mathbb{S}_{2}\right)$. It is a fixed point of the RG equations and is given by $r=1 / 2, r_{A}=-1 / 2, t=1 / 2, t_{A}=1 / 2$ discussed earlier in Sec. 3. Here also $t$ is equal to $t_{A}$ as in the previous case and thus the junction will act like a perfect charge filter resulting in pure SC in the right wire (if spin polarized charge current is injected in the left wire). However, this $\mathbb{S}$-matrix $\left(\mathbb{S}_{2}\right)$ represents an unstable fixed point. Due to the implementation of any small perturbation, the parameters tend to flow away from this unstable fixed point to the most stable disconnected fixed point given by $|r|=1$ as a result of RG flow. So this $\mathbb{S}$-matrix $\left(\mathbb{S}_{2}\right)$ is not a stable operating point for the production of pure SC. Although, it is interesting to note that if we switch on a small perturbation around this fixed point, the charge conductance exhibits a non-monotonic behavior under 
the RG flow (Fig. 12 and also the left diagram of Fig. 17). As discussed earlier, this non-monotonicity results from two competing effects viz., transport through both electron and hole channels and, the RG flow of $g_{1}, g_{2}$. The latter essentially leads to negative differential conductance (NDC) ${ }^{112}$. Elaborating it further, all it means is that if we start an experiment with this given $\mathbb{S}$-matrix $\left(\mathbb{S}_{2}\right)$ at zero temperature and at finite bias, then as we go towards zero bias, the conductance will show a rise with decreasing bias for a certain bias window. This feature can be seen from Fig. 17 . The following aspect of the RG flow can be of direct relevance for manipulating
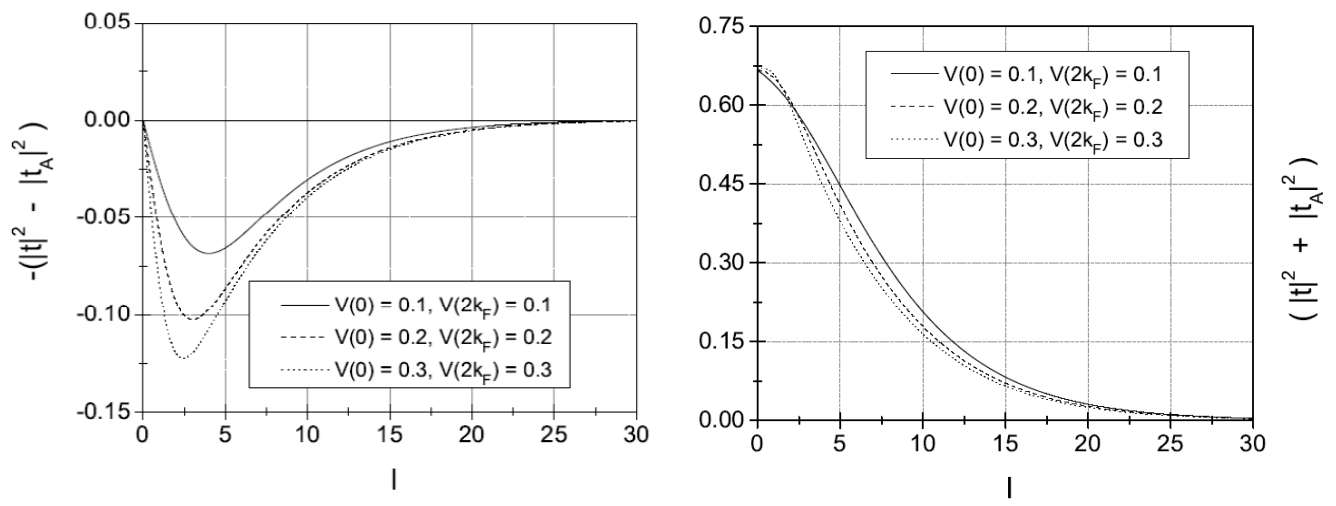

Fig. 18. (Color online) The variation of $-\left(|t|^{2}-\left|t_{A}\right|^{2}\right) \propto G_{\uparrow}^{C}$ or $G_{\downarrow}^{C}$ and the variation of $\left(|t|^{2}+\right.$ $\left.\left|t_{A}\right|^{2}\right) \propto G_{\uparrow}^{S}$ or $G_{\downarrow}^{S}$ are plotted in left and right panel plots as a function of the dimensionless parameter $l$ where $l=\ln (L / d)$ and $L$ is either $L_{T}=\hbar v_{F} / k_{B} T$ at zero bias or $L_{V}=\hbar v_{F} / \mathrm{eV}$ at zero temperature and $d$ is the short distance cut-off for the RG flow. The three curves in each plot correspond to three different values of $V(0)$ and $V\left(2 k_{F}\right)$ for the FSN junction. These plots correspond to the $\mathbb{S}$-matrix given by $\mathbb{S}_{3}$. Figure adapted from Ref. 76 .

in some mesoscopic devices.

Now we switch to the case of FSN junction which comprises of a 1-D ferromagnetic half metal (assuming $\uparrow$ polarization) on one side and a normal 1-D metal on the other side (in a way similar to the set-up shown in Fig. 15). This case is very complicated to study theoretically because the minimal number of independent complex-valued parameters that are required to parameterize the $\mathbb{S}$-matrix is nine as opposed to the previous (symmetric) case which had only four such parameters. These are given by $r_{\uparrow \uparrow}^{11}, r_{\uparrow \uparrow}^{22}, r_{\downarrow \downarrow}^{22}, t_{A \uparrow \uparrow}^{12}, t_{A \downarrow \downarrow}^{21}, r_{A \uparrow \uparrow}^{22}, r_{A \downarrow \downarrow}^{22}, t_{\uparrow \uparrow}^{12}$, and $t_{\uparrow \uparrow}^{21}$. Here, 1 (2) is the wire index for the ferromagnetic (normal) wire while, $\uparrow$ and $\downarrow$ are the respective 
spin polarization indices for the electron. The large number of independent parameters in this case arise because of the presence of ferromagnetic half-metallic wire which destroys both the spin rotation symmetry and the left-right symmetry. The only remaining symmetry is the particle-hole symmetry. Analogous to the RG equations (given by Eqs. 45-48) for the NSN case, it is also possible to write down all the nine RG equations for FSN case and solve them numerically to obtain the results as shown in Fig. 18. In this case, the elements of a representative $\mathbb{S}$-matrix $\left(\mathbb{S}_{3}\right)$ which correspond to the production of pure SC are $\left|r_{\uparrow \uparrow}^{11}\right|=\left|r_{\uparrow \uparrow}^{22}\right|=\left|r_{\downarrow \downarrow}^{22}\right|=\left|t_{A \uparrow \uparrow}^{12}\right|=\left|t_{A \downarrow \downarrow}^{21}\right|=$ $\left|r_{A \uparrow \uparrow}^{22}\right|=\left|r_{A \downarrow \downarrow}^{22}\right|=\left|t_{\uparrow \uparrow}^{12}\right|=\left|t_{\uparrow \uparrow}^{21}\right|=1 / \sqrt{3}$ and the corresponding phases associated with each of these amplitudes are $\pi / 3, \pi, 0,-\pi / 3,0, \pi / 3,0, \pi,-\pi / 3$ respectively. By solving the nine coupled RG equations for the above mentioned nine independent parameters, we have checked numerically that this is not a fixed point of the RG equation and hence it will flow under RG under the application of small perturbation around it and finally reach the trivial stable fixed point given by $r_{\uparrow \uparrow}^{11}=r_{\uparrow \uparrow}^{22}=r_{\downarrow \downarrow}^{22}=1$. Now if we impose a bias on the system from left to right, it will create a pure SC on the right wire because $\left|t_{A \uparrow \uparrow}^{12}\right|$ is exactly equal to $\left|t_{\uparrow \uparrow}^{12}\right|$. On the other hand, of course, this is a highly unstable operating point for production of pure SC as this is not even a fixed point and hence will always flow under any variation of temperature or bias destroying the production of pure SC. In this case also, the spin conductance shows a monotonic behavior while, the charge conductance is non-monotonic and hence will have NDC in some parameter regime. It is worth noticing that in this case the interaction parameters $g_{1}$ and $g_{2}$ both do not scale on the left wire as it is completely spin polarized while $g_{1}$ and $g_{2}$ do scale on the right wire as it is not spin polarized. Hence even if we begin our RG flow with symmetric interaction strengths on both left and right wires, they will develop an asymmetry under the RG flow. The following features are depicted in Fig. 18.

Finally, we consider another important aspect that nicely characterizes these hybrid structures from a spintronics application point of view. If the QW on the two sides of the superconductor are ferromagnetic half metals then we have a junction of FSF. We calculate the tunnelling magnetoresistance ratio (TMR) ${ }^{102}$ which is defined as follows

$$
\mathrm{TMR}=\left[\frac{G_{\uparrow \uparrow}-G_{\uparrow \downarrow}}{G_{\uparrow \downarrow}}\right],
$$

Here, $G_{\uparrow \uparrow}$ corresponds to the conductance across the junction when both left and right wires are in parallel spin-polarized configurations. $G_{\uparrow \downarrow}$ corresponds to the case when the left and right wires are in anti-parallel spin-polarized configurations. Thus, TMR is the maximum relative change in resistance in going from the parallel to the anti-parallel configuration. For the parallel case, the CAR amplitude $\left(t_{A}\right)$ is zero and the only process which contributes to the conductance is the direct tunnelling process. This is because the CAR process involves non-local pairing of $\uparrow \mathrm{e}$ in the left wire with $\downarrow$ e in the right wire to form a Cooper pair. However for $\downarrow$ e, the density of states is zero in the right wire which makes this process completely forbidden. 
Hence, $G_{\uparrow \uparrow} \propto|t|^{2}$. On the other hand, for the anti-parallel case, $G_{\uparrow \downarrow} \propto-\left|t_{A}\right|^{2}$ as there is no density of states for the $\uparrow \mathrm{e}$ in the right lead and so no direct tunnelling of $\uparrow \mathrm{e}$ across the junction is allowed; hence CAR is the only allowed process. Note that the negative sign in $G_{\uparrow \downarrow}$ leads to a very large enhancement of TMR (as opposed to the case of standard ferromagnet-normal metal-ferromagnet (FNF) junction) since the two contributions will add up. A related set-up has been studied in ${ }^{105}$ where also a large TMR has been obtained.

One can then do the RG analysis for both parallel and anti-parallel FSF cases. It turns out that the equations for $|t|$ and $\left|t_{A}\right|$ are identical leading to identical temperature (bias) dependance. The RG equation for $\left|t_{A}\right|$ is

$$
\frac{d t_{A}}{d l}=-\beta t_{A}\left[1-\left|t_{A}\right|^{2}\right],
$$

Here, $\beta=\left(g_{2}-g_{1}\right) / 2 \pi \hbar v_{F}$. Also $|t|$ satisfies the same equation. So, in a situation where the reflection amplitudes at the junction for the two cases are taken to be equal then it follows from Eq. 73 that the TMR will be pinned to its maximum value i.e. magnitude of TMR $=2$ and the temperature dependence will be flat even in the presence of e-e interactions inside the QW. The latter feature can be very useful in fabricating spintronic devices based on FSF junctions of 1-D QWs.

\section{Summary and Outlook}

To summarize, in this review we have presented the effects of e-e interactions on transport properties of mesoscopic hybrid structures which include superconducting junctions of multiple 1-D QWs. We start with an elementary introduction to the technique called weak interaction renormalization group (WIRG) used to tackle e-e interactions inside the QW in this review. Within this approach one can obtain the RG equations for the effective $\mathbb{S}$-matrix and calculate the various fixed point $\mathbb{S}$-matrices representing the junction. Then we have generalized the WIRG approach to study transport through a superconducting junction of multiple 1-D interacting QWs. Applying the WIRG approach we have derived the RG equations for the effective $\mathbb{S}$-matrix and obtained the various fixed point $\mathbb{S}$-matrices representing the superconducting junction. Our analysis led to the finding of a novel fixed point with intermediate AR and CAR named SFP which turns out to be unstable and hence experimentally hard to access. We also compute the length scale (or temperature) dependance of the Landauer-Buttiker conductance taking into account e-e interaction induced forward and back-scattering processes. The conductance shows a non-monotonic behaviour around the SFP even for the case of two-wire superconducting junction (NSN) in contrast to the NS junction where the dependence is purely monotonic. When more than two wires are attached to the superconductor, we found even more exotic fixed points like the AGFP which turns out to be a stable fixed point for a reflection-less $(r=0)$ symmetric junction. Thus it would be an interesting experimental challenge to look for signature of the AGFP at intermediate temperatures. 
As the next step, we have laid down a scheme to perform a systematic stability analysis which works well for both normal and superconducting junctions of multiple LL QW. We compute the power laws associated with the renormalization group flow around the various fixed points of this system using the generators of the $S U(3)$ and $S U(4)$ group to generate the appropriate parameterization of a $\mathbb{S}$-matrix representing small deviations from a given fixed point $\mathbb{S}$-matrix. Using our procedure, we reproduce the known power laws for the GFP of a three-wire junction. Then we apply the same precedure to a superconducting junction of two LL wires and obtain the non-trivial power laws around the SFP which are non-linear functions of $V(0)$ and $V\left(2 k_{F}\right)$. Finally, we calculate the Landauer-Buttiker conductance associated with the perturbations switched on around these fixed points and found the explicit voltage or temperature power law dependence.

From the application point of view we demonstrate possible scenarios for production of pure SC and large TMR ratios from CT and CAR processes across a superconducting junction comprising of 1-D QWs. In particular we have studied both spin and charge transport in NSN, FSN, and FSF structures in the context of 1-D QW. We show that there are fixed points in the theory which correspond to the production of pure SC. We describe the effect of e-e interaction and note the stability of the production of pure SC against temperature and voltage variations. Finally, we also show that the presence of the CAR process heavily enhances the TMR in such geometries and also calculate its power law temperature dependence.

Before we conclude, it is worth mentioning that the geometry discussed in this review is also of direct interest for the production of non-local entangled electron pairs propagating in two different wires. These electron pairs are produced by Cooper pair splitting via CAR processes when the superconductor is biased with respect to the wires comprising the junction. One can then ask if e-e interaction in the QWs actually leads to enhancement of entangled electron pair production via the CAR processes. For example, we have observed that for the NSN junction with $r=0, t=0, r_{A} \neq 0, t_{A} \neq 0$, interaction can lead to enhancement of the CAR amplitude $\left(t_{A}\right)$ under the RG flow. The latter scenario implies that for the case when the superconductor is biased with respect to the wires, inter-electron interactions enhance the production of non-local entangled pairs, for which the amplitude $t_{A}$ is relevant, as compared to the local entangled pairs for which the amplitude $r_{A}$ would be relevant. This is consistent with the results of Recher and Loss ${ }^{106,107}$ who also argued that it is energetically more favourable for the two entangled electrons of the Cooper pair to go into different wires, rather than the same wire. Finally the RG flow leads to a fixed point with $t_{A}=1$ where the system becomes a perfect entangler. A more general case would be when $r \neq 0, t \neq 0, r_{A} \neq 0, t_{A} \neq 0$. To study this case, one can start from the two wires SFP $\mathbb{S}$-matrix and study the RG flow of $t_{A}$ for an $\mathbb{S}$-matrix which is in the close vicinity of this fixed point. The result of this study is shown in Fig. 12. We show that starting from the short-distance cut-off $d$ the RG flow initially leads to enhancement of $t_{A}$ which will lead to an enhancement in the production of non-local entangled pairs. Hence, these studies establish the fact 
e-e interactions inside the QWs can actually lead to an enhancement of non-local entangled electron pair production. The above mentioned scenario of production of entangled electron pairs in Cooper pair beam splitter geometry has been put forwarded very recently via the differential conductance and shot noise charge as well as spin cross-correlation measurements ${ }^{53,54,57,114}$.

\section{Acknowledgements}

We would like to thank our collaborators Sourin Das and Sumathi Rao. We especially thank Sumathi Rao for careful reading of the manuscript and valuable comments. This work is supported by the Swiss NSF, NCCR Nanoscience, and NCCR QSIT.

\section{Appendix A. RG equations for FSN junction}

Here we present the RG equations for nine independent parameters in case of a FSN junction.

$$
\begin{aligned}
\frac{d r}{d l} & =\left[\frac{\beta}{2} r\left(1-|r|^{2}\right)-\frac{\alpha}{2}\left(t r^{\prime \star} t^{\prime}+r^{\prime \prime \star} t_{A} t_{A}^{\prime}\right)+\frac{\alpha}{2}\left(t r_{A}^{\star} t_{A}^{\prime}+t_{A} r_{A}^{\prime \star} t^{\prime}\right)\right] \text { (A.1) } \\
\frac{d t}{d l} & =-\left[\frac{\beta}{2}|r|^{2} t+\frac{\alpha}{2}\left(\left|r^{\prime}\right|^{2} t+t_{A} r^{\prime \prime \star} r_{A}^{\prime}\right)-\frac{\alpha^{\prime}}{2}\left(r_{A}^{\star} r_{A}^{\prime} t+r^{\prime} r_{A}^{\prime \star} t_{A}\right)\right], \quad \text { (A.2) } \\
\frac{d t_{A}}{d l} & =-\left[\frac{\beta}{2}|r|^{2} t_{A}+\frac{\alpha}{2}\left(\left|r^{\prime \prime}\right|^{2} t_{A}+t r^{\prime \star} r_{A}\right)-\frac{\alpha^{\prime}}{2}\left(r_{A}^{\star} r^{\prime \prime} t+r_{A} r_{A}^{\prime \star} t_{A}\right)\right], \text { (A.3) } \\
\frac{d r^{\prime}}{d l} & =-\left[\frac{\beta}{2} r^{\star} t t^{\prime}+\frac{\alpha}{2}\left[r^{\prime \prime \star} r_{A} r_{A}^{\prime}-r^{\prime}\left(1-\left|r^{\prime}\right|^{2}\right)\right]-\frac{\alpha^{\prime}}{2} r^{\prime}\left(r_{A} r_{A}^{\prime \star}+r_{A}^{\star} r_{A}^{\prime}\right)\right], \quad \text { (A.4) } \\
\frac{d r_{A}}{d l} & =-\left[\frac{\beta}{2} r^{\star} t t_{A}+\frac{\alpha}{2} r_{A}\left(\left|r^{\prime}\right|^{2}+\left|r^{\prime \prime}\right|^{2}\right)+\frac{\alpha^{\prime}}{2}\left(r_{A}-r_{A}^{2} r_{A}^{\prime \star}-r_{A}^{\star} r^{\prime} r^{\prime \prime}\right)\right], \quad \text { (A.5) } \\
\frac{d t^{\prime}}{d l} & =-\left[\frac{\beta}{2}|r|^{2} t^{\prime}+\frac{\alpha}{2}\left(\left|r^{\prime}\right|^{2} t^{\prime}+r_{A} r^{\prime \prime \star} t_{A}^{\prime}\right)-\frac{\alpha^{\prime}}{2}\left(r_{A} r_{A}^{\prime \star} t^{\prime}+r^{\prime} r_{A}^{\star} t_{A}^{\prime}\right)\right], \\
\frac{d r^{\prime \prime}}{d l} & =-\left[\frac{\beta}{2} r^{\star} t_{A} t_{A}^{\prime}+\frac{\alpha}{2}\left[r^{\prime \star} r_{A} r_{A}^{\prime}-r^{\prime \prime}\left(1-\left|r^{\prime \prime}\right|^{2}\right)\right]-\frac{\alpha^{\prime}}{2} r^{\prime \prime}\left(r_{A} r_{A}^{\prime \star}+r_{A}^{\star} r_{A}^{\prime}(\mathrm{A}) \text {. } .7\right)\right. \\
\frac{d r_{A}^{\prime}}{d l} & =-\left[\frac{\beta}{2} r^{\star} t_{A}^{\prime} t+\frac{\alpha}{2} r_{A}^{\prime}\left(\left|r^{\prime \prime}\right|^{2}+\left|r^{\prime}\right|^{2}\right)+\frac{\alpha^{\prime}}{2}\left(r_{A}^{\prime}-r_{A}^{\star} r_{A}^{\prime 2}-r_{A}^{\prime \star} r^{\prime} r^{\prime \prime}\right)\right] \text { (A. .8) } \\
\frac{d t_{A}^{\prime}}{d l} & =-\left[\frac{\beta}{2}|r|^{2} t_{A}^{\prime}+\frac{\alpha}{2}\left(\left|r^{\prime \prime}\right|^{2} t_{A}^{\prime}+r^{\prime \star} t^{\prime} r_{A}^{\prime}\right)-\frac{\alpha^{\prime}}{2}\left(r_{A}^{\prime \star} r^{\prime \prime} t^{\prime}+r_{A}^{\prime} r_{A}^{\star} t_{A}^{\prime}\right)\right] \text { (A.9) }
\end{aligned}
$$




\section{Appendix B. RG equations for 3 wire NSN junction}

Here we give the RG equations for six independent parameters in case of a symmetric 3 wire NSN junction.

$$
\begin{aligned}
\frac{d r}{d l} & =-\left[\frac{\alpha}{2}\left[r^{\star}\left(r_{A}^{2}+t^{2}+t^{\prime 2}+t_{A}^{2}+t_{A}^{\prime 2}\right)-r\left(1-|r|^{2}\right)\right]-\alpha^{\prime}\left[r\left|r_{A}\right|^{2}+r_{A}^{\star}\left(t t_{A}+t^{\prime} t_{A}^{\prime}\right)\right]\right], \quad \text { (B.1) } \\
\frac{d t}{d l} & =-\left[\alpha\left[|r|^{2} t+r^{\star}\left(r_{A} t_{A}+t^{\prime 2}+t_{A}^{\prime 2}\right)\right]-\alpha^{\prime}\left[\left|r_{A}\right|^{2} t+r_{A}^{\star}\left(r t_{A}+t^{\prime} t_{A}^{\prime}\right)\right]\right], \\
\frac{d t^{\prime}}{d l} & =-\left[\frac{\alpha}{2}\left[2|r|^{2} t^{\prime}+r^{\star}\left(t t^{\prime}+t_{A} t_{A}^{\prime}+2 r_{A} t_{A}^{\prime}\right)\right]-\frac{\alpha^{\prime}}{2}\left\{2\left|r_{A}\right|^{2} t^{\prime}+r_{A}^{\star}\left[r\left(t^{\prime}+t_{A}^{\prime}\right)+\left(t^{\prime} t_{A}+t t_{A}^{\prime}\right) \mid \beta\right]\right] 3 .\right) \\
\frac{d r_{A}}{d l} & =-\left[\frac{\alpha}{2}\left\{2|r|^{2} r_{A}+r^{\star}\left[2 t t_{A}+t_{A}^{\prime}\left(t+t^{\prime}\right)\right]\right\}+\frac{\alpha^{\prime}}{2}\left[r_{A}-r_{A}^{\star}\left(r^{2}+r_{A}^{2}+t_{A}^{2}+t_{A}^{\prime 2}+2 t t^{\prime}\right)\right]\right], \\
\frac{d t_{A}}{d l} & =-\left[\frac{\alpha}{2}\left[2\left(|r|^{2} t_{A}+r^{\star} r_{A} t\right)+r^{\star} t^{\prime} t_{A}^{\prime}+r_{A}^{\star} t^{\prime 2}\right]-\frac{\alpha^{\prime}}{2}\left[2\left(\left|r_{A}\right|^{2} t_{A}+r t r_{A}^{\star}\right)+r_{A}^{\star}\left(t^{\prime 2}+t_{A}^{\prime 2}\right)\right]\right],(\mathrm{B} .5) \\
\frac{d t_{A}^{\prime}}{d l} & =-\left[\frac{\alpha}{2}\left[2\left(|r|^{2} t_{A}^{\prime}+r^{\star} r_{A} t^{\prime}\right)+r^{\star}\left(t^{\prime} t_{A}+t t_{A}^{\prime}\right)\right]-\frac{\alpha^{\prime}}{2}\left[2\left(\left|r_{A}\right|^{2} t_{A}^{\prime}+r t^{\prime} r_{A}^{\star}\right)+r_{A}^{\star}\left(t t^{\prime}+t_{A} t_{A}^{\prime}\right)\right]\right](\mathrm{B} .6)
\end{aligned}
$$

\section{References}

1. A. Yacoby, H. L. Stormer, N. S. Wingreen, L. N. Pfeiffer, K.W. Baldwin, and K.W. West, Phys. Rev. Lett 77(22), 4613 (1996).

2. O. M. Auslaender, A. Yacoby, R. Picciotto, L. N. Pfeiffer, K. W. Baldwin, and K. W. West, Phys. Rev. Lett 84, 1764 (2000).

3. R. Picciotto, L. N. Pfeiffer, K. W. Baldwin, and K. W. West, Phys. Rev. Lett 92, 36805 (2004)

4. O. M. Auslaender, A. Yacoby, R. de Picciotto, K. W. Baldwin, L. N. Pfeiffer, and K. W. West, Science 295, 825 (2002).

5. O. Auslaender, H. Steinberg, A. Yacoby, Y. Tserkovnyak, B. Halperin, K. Baldwin, L. Pfeiffer, and K. W. West, Science 308, 88 (2005).

6. C. Papadopoulos, A. Rakitin, J. Li, A. S. Vedeneev, and J. M. Xu, Phys. Rev. Lett. 85(16), 3476 (2000).

7. C. L. Kane and M. P. A. Fisher, Phys. Rev. B 46, 15233 (1992).

8. S. Tarucha, T. Honda, and T. Saku, Sol. St. Comm. 94, 413 (1995).

9. C. T. Liang, M. Pepper, M. Y. Simmons, C. G. Smith, and D. A. Ritchie, Phys. Rev. $B$ 61, 9952 (2000).

10. B. E. Kane, G. R. Facer, A. S. Dzurak, N. E. Lumpkin, R. G. Clark, L. N. Pfeiffer, and K. W. West, App. Phys. Lett. 72, 3506 (1998).

11. D. J. Reilly, G. R. Facer, A. S. Dzurak, B. E. Kane, R. G. Clark, P. J. Stiles, J. L. OBrien, N. E. Lumpkin, L. N. Pfeiffer, and K. W. West, Phys. Rev. B 63, 121311(R) (2001).

12. S. I. and H. J. Schulz, Phys. Rev. B 52, R17040 (1995).

13. D. L. Maslov and M. Stone, Phys. Rev. B 52, R5539 (1995).

14. V. V. Ponomarenko, Phys. Rev. B 52, R8666 (1995).

15. S. I. and H. J. Schulz, Phys. Rev. B 59, 3040 (1999).

16. D. L. Maslov, Phys. Rev. B 52, R14368 (1995).

17. A. Furusaki and N. Nagaosa, Phys. Rev. B 54, R5239 (1996).

18. S. Lal, S. Rao, and D. Sen, Phys. Rev. Lett. 87(2), 026801 (2001).

19. C. Nayak, M. P. A. Fisher, A. W. W. Ludwig, and H. H. Lin, Phys. Rev. B 59, 15694 (1999). 
20. M. Oshikawa, C. Chamon and I. Affleck, J. Stat. Mech. 02, P02008 (2006)

21. T. Giamarchi, Quantum physics in one dimension (Oxford Science Publications, 2001).

22. A. O. Gogolin, A. A. Nersesyan, and A. M. Tsvelik, Bosonization and Strongly Correlated Systems (Cambridge University Press, Cambridge, 1998).

23. P. Kopietz, Bosonization of interacting fermions in arbitary dimensions (Springer).

24. J. von Delft and H. Schoeller, Ann. Phys. 7, 225 (1998).

25. S. Rao and D. Sen, Lectures on bosonisation in Field Theories in Condensed Matter physics, (Hindustan Book Agency, New Delhi, 2001).

26. D. Yue, L. I. Glazman, and K. A. Matveev, Phys. Rev. B 49, 1966 (1994).

27. S. Lal, S. Rao, and D. Sen, Phys. Rev. B 66, 165327 (2002).

28. D. G. Polyakov and I. Gornyi, Phys. Rev. B 68, 035421 (2003).

29. D. N. Aristov and P. Wolfle, Euro. Phys. Lett. 82, 27001 (2008).

30. D. N. Aristov and P. Wolfle, Phys. Rev. B 80, 045109 (2009).

31. M. Bockrath, D. H. Cobden, J. Lu, A. G. Rinzler, L. Balents, and P. L. McEuen, Nature 397, 598 (1999).

32. A. F. Andreev, Sov. Phys. JETP 19, 1228 (1964).

33. J. Bardeen, L. N. Cooper, and J. R. Schrieffer, Phys. Rev. 108, 1175 (1957).

34. P. G. de Gennes, Superconducitivity of Metals and Alloys, (Addison-Wesley Publishing Co., Reading, MA, 1989).

35. G. E. Blonder, M. Tinkham, and T. M. Klapwijk, Phys. Rev. B 25, 4515 (1982).

36. S. Russo, M. Kroug, T. M. Klapwijk, and A. F. Morpurgo, Phys. Rev. Lett. 95, $027002(2005)$.

37. D. Beckmann, H. B. Weber, and H. V. Löhneysen, Phys. Rev. Lett. 93(19), 197003 (2004).

38. A. Kleine, A. Baumgartner, J. Trbovic, and C. Schonenberger, Europhys. Lett. 87, 27011 (2009).

39. A. F. Morpurgo, J. Kong, C. M. Marcus, and H. Dai, Science 286, 263 (1999).

40. G. Falci, D. Feinberg, and F. W. J. Hekking, Europhys. Lett. 54, 255 (2001).

41. G. Bignon, M. Houzet, F. Pistolesi, and F. W. J. Hekking, Europhys. Lett. 67, 110 (2004).

42. P. Samuelsson and M. Büttiker, Phys. Rev. B 66, 201306 (2002).

43. P. Samuelsson and M. Büttiker, Phys. Rev. Lett. 89, 046601 (2002).

44. P. Samuelsson, E. V. Sukhorukov, and M. Büttiker, Phys. Rev. Lett. 91, 157002 (2003).

45. P. Recher, E. V. Sukhorukov, and D. Loss, Phys. Rev. B 63, 165314 (2001).

46. V. Bouchiat, N. Chtchelkatchev, D. Feinberg, G. B. Lesovik, T. Martin, and J. Torres, Nanotechnology 14, 77 (2003).

47. A. L. Yeyati, F. S. Bergeret, A. Martin-Rodero, and T. M. Klapwijk, Nat. Phys. 3, 455 (2007).

48. J. Torres, and T. Martin, Eur. Phys. J. B 12, 319 (1999).

49. G. B. Lesovik, T. Martin, and G. Blatter, Eur. Phys. J. B 24, 287 (2001).

50. R. Melin, C. Benjamin, and T. Martin, Phys. Rev. B 7794512 (2008).

51. P. Cadden-Zimansky, and V. Chandrasekhar, Phys. Rev. Lett. 97, 237003 (2006).

52. D. Chevallier, J. Rech, T. Jonckheere, and T. Martin, Phys. Rev. B 83, 125421 (2011).

53. J.Wei, and V. Chandrasekhar, Nat. Phys. 4, 494 (2010).

54. L. Hofstetter, S. Csonka, J. Nygard, and C. Schonenberger, Nature 461960 (2009).

55. L. G. Herrmann, F. Portier, P. Roche, A. L. Yeyati, T. Kontos, and C. Strunk, Phys. Rev. Lett. 104, 026801 (2010). 
56. L. Hofstetter et. al, Phys. Rev. Lett. 107, 136801 (2011).

57. A. Das, Y. Ronen, M. Heiblum, D. Mahalu, A. V. Kretinin, and H. Shtrikman, Nat. Commun. 31165 (2012).

58. T. Takane and Y. Koyama, J. Phys. Soc. Jpn. 65, 3630 (1996).

59. T. Takane and Y. Koyama, J. Phys. Soc. Jpn. 66, 419 (1997).

60. R. Fazio, F. W. J. Hekking, A. A. Odintsov, and R. Raimondi, Superlattices Microstruct. 25, 1163 (1999).

61. M. Titov, M. Muller, and W. Belzig, Phys. Rev. Lett. 97, 237006 (2006).

62. S. Vishveshwara, C. Bena, L. Balents, and M. P. A. Fisher, Phys. Rev. B 66, 165411 (2002).

63. D. Maslov, M. Stone, P. Golbert and D. Loss, Phys. Rev. B 53 (3), 1548 (1996).

64. S. Das, and S. Rao, Phys. Rev. B 78, 205421 (2008).

65. S. Das, S. Rao, and D. Sen, Phys. Rev. B 70, 085318 (2004).

66. F. D. M. Haldane, Jour. Phys. C 14, 2585 (1981).

67. K. A. Matveev, D. Yue, and L. I. Glazman, Phys. Rev. Lett. 71, 3351 (1993).

68. J. Sólyom, Adv. Phys. 28, 201 (1979).

69. P. W. Anderson, J. Phys. C 3, 2436 (1970).

70. C. Chamon, M. Oshikawa, and I. Affleck, Phys. Rev. Lett. 91, 206403 (2003).

71. S. Das, S. Rao, and D. Sen, Phys. Rev. B 74, 045322 (2006).

72. V. R. Chandra, S. Rao, and D. Sen, Europhys. Lett. 75, 797 (2006).

73. V. R. Chandra, S. Rao, and D. Sen, Phys. Rev. B 75, 045435 (2007).

74. T. Takane, J. Phys. Soc. Jpn. 66, 537 (1997).

75. S. Das, S. Rao, and A. Saha, Phy. Rev. B 77, 155418 (2008).

76. S. Das, S. Rao, and A. Saha, Europhys. Lett. 81, 67001 (2008).

77. S. Das, S. Rao, and A. Saha, Phy. Rev. B 79, 155416 (2009).

78. B. Bellazzini, M. Mintchev, and P. Sorba, J. Phys. A 40, 2485 (2007).

79. B. Bellazzini, P. Calabrese, and M. Mintchev, Phys. Rev. B 79, 085122 (2009).

80. B. Bellazzini, M. Burrello, M. Mintchev, and P. Sorba Proc. Symp. Pure Math. 77, 639 (2008).

81. B. Bellazzini, M. Mintchev, and P. Sorba Phys. Rev. B 80, 245441 (2008).

82. B. Bellazzini, M. Mintchev, and P. Sorba Phys. Rev. B 82, 195113 (2010).

83. D. N. Aristov, A. P. Dmitriev, I.V. Gornyi, V.Yu. Kachorovskii, D. G. Polyakov, and P. Wölfle Phys. Rev. Lett. 105, 266404 (2010).

84. D. N. Aristov Phys. Rev. B 83, 115446 (2011).

85. D. N. Aristov and P. Wölfle Phys. Rev. B 84, 155426 (2011).

86. D. N. Aristov and P. Wölfle Phys. Rev. B 86, 035137 (2012).

87. D. N. Aristov and P. Wölfle arXiv:1304.5167 (2013).

88. Yu. V Nazarov and L. I. Glazman Phys. Rev. Lett. 91, 126804 (2003).

89. A. Agarwal, S. Das, S. Rao, and D. Sen Phys. Rev. Lett. 103, 026401 (2009).

90. A. Agarwal, S. Das, and D. Sen Phys. Rev. B 81, 035324 (2010).

91. C. Chamon, M. Oshikawa, and I. Affleck Phys. Rev. Lett. 91, 206403 (2003).

92. J. S. Griffith Trans. Faraday Soc. 49, 345 (1953).

93. J. Brauer, F. Hübler, M. Smetanin, D. Beckmann, and H. v. Löhneysen Phys. Rev. B 81, 024515 (2010).

94. P. Cadden-Zimansky, J.Wei and V. Chandrasekhar Nat. Phys. 5, 393 (2009).

95. D. Futterer, M. Governale, M. G. Pala, J. König Phys Rev B 79, 054505 (2009).

96. J. Eldridge, M. G. Pala, M. Governale, J. König Phys. Rev. B 82, 184507 (2010).

97. A. Braggio, M. Governale, M. G. Pala, J. König Solid State Comm. 151, 155 (2011).

98. A. G. Moghaddam, M. Governale, J. König Phys. Rev. B 85, 094518 (2012).

99. V. Meden, S. Andergassen, T. Enss, H. Schoeller, and K. Schoenhammer, New Jour- 
nal of Physics 10, 045012 (2008).

100. D. Sen, The power law was confirmed to be $-\alpha / 3$, (private communication).

101. S. Datta and B. Das, Appl. Phys. Lett. 56, 665 (1990).

102. I. Zutik, J. Fabian, and S. D. Sharma, Rev. Mod. Phys. 76, 323 (2004).

103. E. I. Rashba, Physica E 34, 31 (2006).

104. S. K. Watson, R. M. Potok, C. M. Marcus, and V. Umansky, Phys. Rev. Lett. 91, 258301 (2003).

105. F. Giazotto, F. Taddei, F. Beltram, and R. Fazio, Phys. Rev. Lett. 97, 087001 (2006).

106. P. Recher and D. Loss, J. Supercond. Novel Magn. 15, 49 (2002).

107. P. Recher and D. Loss, Phys. Rev. B 65, 165327 (2002).

108. S. Das, S. Rao and A. Saha, Eur. Phys. J. B 72, 139 (2009).

109. A. Kundu, S. Rao and A. Saha, Europhys. Lett. 88, 57003 (2009).

110. D. Futterer, M. Governale, and J. König, Europhys. Lett. 91, 47004 (2010).

111. A. Cottet, and W. Belzig, Phys. Rev. B 77, 064517 (2008).

112. L. Esaki, Phys. Rev. 109, 603 (1958).

113. M. J. Wolf, F. Hübler, S. Kolenda, H. v. Löhneysen, and D. Beckmann, Phys. Rev. $B$ 87, 024517 (2013).

114. T. Noh, M. Houzet, J. S. Meyer, and V. Chandrasekhar, arXiv:1304.0669 (2013). 